\title{
NONLINEAR ANALYSIS OF EEG FOR EPILEPTIC SEIZURES
}

\author{
L. M. Hively \\ N. E. Clapp \\ C. S. Daw \\ W.F. Lawkins \\ Oak Ridge National Laboratory \\ and \\ M. L. Eisenstadt \\ Knoxville Neurology Clinic
}

April 1995

DISTRIBUTION OF THIS DOCUMENT IS URLRTIED 


\section{DISCLAIMER}

This report was prepared as an account of work sponsored by an agency of the United States Government. Neither the United States Government nor any agency thereof, nor any of their employees, make any warranty, express or implied, or assumes any legal liability or responsibility for the accuracy, completeness, or usefulness of any information, apparatus, product, or process disclosed, or represents that its use would not infringe privately owned rights. Reference herein to any specific commercial product, process, or service by trade name, trademark, manufacturer, or otherwise, does not necessarily constitute or imply its endorsement, recommendation, or favoring by the United States Government or any agency thereof. The views and opinions of authors expressed herein do not necessarily state or reflect those of the United States Government or any agency thereof. 


\section{DISCLAIMER}

Portions of this document may be illegible in electronic image products. Images are produced from the best available original document. 
ORNL/TM-12961

\title{
NONLINEAR ANALYSIS OF EEG FOR EPILEPTIC SEIZURES
}

\author{
L. M. Hively \\ N. E. Clapp \\ C. S. Daw \\ W. F. Lawkins \\ Oak Ridge National Laboratory \\ and \\ M. L. Eisenstadt \\ Knoxville Neurology Clinic \\ St. Mary's Medical Center \\ 800 E. Oak Hill Avenue, N.E. \\ Knoxville, TN 37917 \\ April 1995 \\ Prepared for \\ Laboratory Directed Research and \\ Development Program \\ DOE Activity No. 473 \\ Prepared by the \\ Engineering Technology Division \\ Oak Ridge National Laboratory \\ Oak Ridge, TN 37831-7294 \\ managed by \\ MARTIN MARIETTA ENERGY SYSTEMS, INC. \\ for the \\ U.S. DEPARTMENT OF ENERGY \\ under contract DE-AC05-84OR21400
}

The submitted manuscript has been authored by a contractor of the U.S. Government under contract No. DE-AC05-84OR21400. Accordingly, the U.S. Government retains a nonexclusive, royalty-free license to publish or reproduce the published form of this contribution, or allow others to do so, for U.S. Government purposes. 
$\square$

. 


\section{CONTENTS}

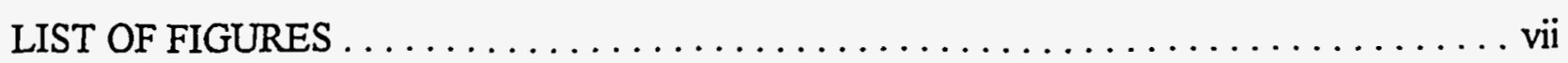

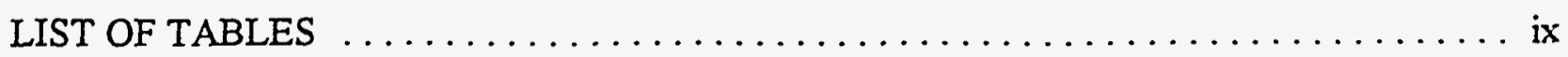

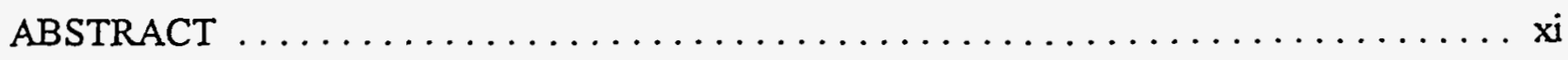

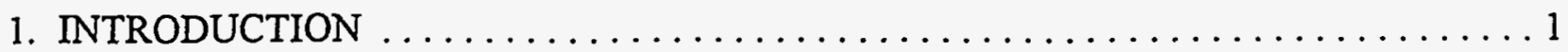

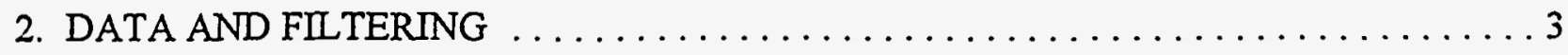

3. NONLINEAR ANALYSIS TOOLS FOR EEG DATA $\ldots \ldots \ldots \ldots \ldots \ldots \ldots$

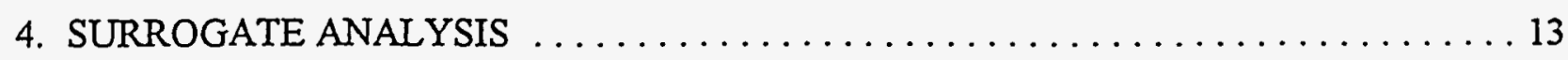

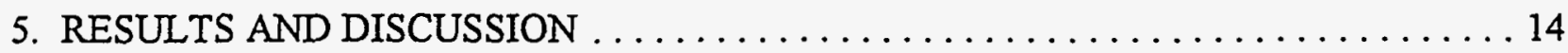





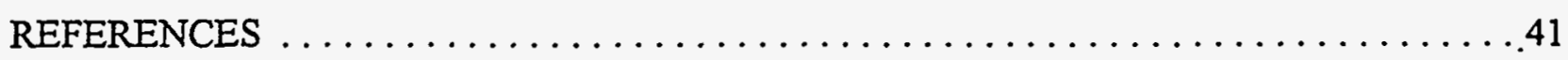

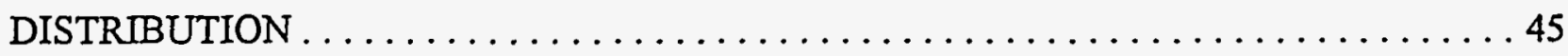





\section{LIST OF FIGURES}

Fig. 2.1: $\quad$ EEG electrode positions on the patient's scalp $\ldots \ldots \ldots \ldots \ldots \ldots \ldots$

Fig. 2.2: $\quad$ Sample plots of non-seizure EEG data $\ldots \ldots \ldots \ldots \ldots \ldots \ldots$

Fig. 5.1: $\quad$ Results for raw EEG (e-) data for dataset $\# 73317 \ldots \ldots \ldots \ldots \ldots \ldots$

Fig. 5.2: $\quad$ Results for artifact EEG data for dataset $\# 73317 \ldots \ldots \ldots \ldots \ldots \ldots \ldots$

Fig. 5.3: $\quad$ Results for artifact-filtered EEG data dataset $\# 73317 \ldots \ldots \ldots \ldots$

Fig. 5.4: $\quad$ Results for artifact- and low-pass-filtered EEG data for dataset $\# 73317 \ldots 21$

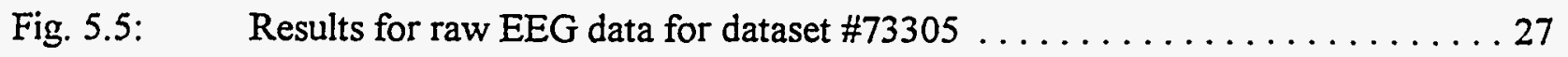

Fig. 5.6: $\quad$ Results for artifact EEG data for dataset $\# 73305$. . . . . . . . . . . . . . 29

Fig. 5.7: $\quad$ Results for artifact-filtered EEG data for dataset $\# 73305 \ldots \ldots \ldots \ldots$

Fig. 5.8: $\quad$ Results for artifact- and low-pass-filtered EEG data for dataset \#73305 . . . 33

vii 
. 


\section{LIST OF TABLES}

Table $4.1 \quad$ Surrogate results for dataset $\# 73305 \ldots \ldots \ldots \ldots \ldots \ldots \ldots$

Table 5.1 Summary of seizure indicators in dataset $\# 73317 \ldots \ldots \ldots \ldots \ldots$

Table $5.2 \quad$ Ratios of averages and standard deviations for dataset $\# 73317 \ldots \ldots$

Table $5.3 \quad$ Summary of seizure indicators in dataset $\# 73305 \ldots \ldots \ldots \ldots \ldots$

Table $5.4 \quad$ Ratios of averages and standard deviations for dataset $\# 73305 \ldots \ldots \ldots$ 


\begin{abstract}
We apply chaotic time series analysis (CTSA) to human electroencephalogram (EEG) data. Three epoches were examined: epileptic seizure, non-seizure, and transition from non-seizure to seizure. The CTSA tools were applied to four forms of these data: raw EEG data (e-data), artifact data (f-data) via application of a quadratic zero-phase filter of the raw data, artifact-filtered data (g-data) that was the residual after subtracting f-data from e-data, and a low-pass-filtered version (h-data) of g-data. Two different seizures were analyzed for the same patient. Several nonlinear measures uniquely indicate an epileptic seizure in both cases, including an abrupt decrease in the time per wave cycle in $\mathrm{f}$-data, an abrupt increase in the Kolmogorov entropy and in the correlation dimension for $\mathrm{e}-\mathrm{h}$ data, and an abrupt increase in the correlation dimension for e-h data. The transition from normal to seizure state also is characterized by distinctly different trends in the nonlinear measures for each seizure and may be potential seizure predictors for this patient. Surrogate analysis of e-data shows that statistically significant nonlinear structure is present during the non-seizure, transition, and seizure epoches.
\end{abstract}





\section{INTRODUCTION}

The theory of nonlinear dynamics provides a basis for understanding and potentially controlling many complex physical and engineering systems. An extensive literature exists for nonlinear dynamics in the brain and related work [1-51]. It is well known that brain waves exhibit seemingly random, unpredictable behavior, that is characteristic of deterministic chaos $[2,5,8,9$, $23,28,35,42,49,50]$. Early work by Cartwright and Littlewood [6] and by Levinson [21] showed that systems of nonlinear differential equations are representative of self-excitatory biological processes, such as neural discharge, and have chaotic orbits. Moreover, chaotic behavior is "normal," while nonchaotic or periodic behavior is indicative of pathophysiology in experimental epilepsy [47], in EEG behavior of the human brain in the presence of neurodegenerative disorders [1,3], and in EEG changes with aging [22]. Recent work [38] showed that chemically-induced seizures in rat-brain can be electrically controlled, leading to speculation $[7,14,24,26]$ that human epilepsy may be controlled without drug or surgical intervention. However, effective use of chaos control for epilepsy requires definitive seizure prediction. Thus, the present work analyzes human EEG data via chaotic time series analysis (CTSA) methods to predict the onset of an epileptic seizure.

Nonlinear analysis of neurological diseases via EEG data is extensive. For example, see the 1992 review by Pritchard and Duke [31]. Epilepsy can be recognized only with clear EEG manifestations, but even these seizures are not easy to detect because there is no stereotyped pattern characteristic of all seizures [15]. Work by Olsen and colleagues [25] used various linear measures with autoregressive modeling, discriminant analysis, clustering, and artificial neural networks. Valuable nonlinear tools for studying EEG data include correlation dimension [10, 27, 43], mutual information function [10, 27], Kolmogorov entropy [17], and phase-space attractors [16]. CTSA of epileptic seizures [12] found clear evidence of chaos in the EEG data via correlation dimension and largest Lyapunov exponent. Fractal models have been used to describe the generation of action potentials in neurons [46]. Electrical signals from cat and rat brain demonstrated strong determinism for potassium-induced seizure-like states, while non-seizure states were stochastic [37]. Krystal [19] found neuronal hypersynchrony in electroconvulsively induced seizures, as measured by EEG amplitude, coherence, and the largest Lyapunov exponent. However, we are unaware of any work that applies several CTSA measures to EEG data for systematic characterization of non-seizure, seizure, and transition-to-seizure.

Very recent analysis by Theiler [48] studied correlation dimension and Lyapunov exponent, using a form of surrogate analysis on a single EEG time series during an epileptic seizure. The surrogate analysis involved a random shuffling of blocks of time serial data, each block containing one quasi-periodic spike-wave complex. The auto-correlation function for the original data is nearly indistinguishable from the surrogate data. The correlation dimension for the original data is significantly different from the surrogate data only at large scale sizes and large embedding dimensions. The maximum Lyapunov exponent $(\lambda)$ was negative for both the original and surrogate data and not substantially different, contrary to previous work which found positive $\lambda$ values. Theiler concluded that his analysis suggests a nonlinear oscillator with noise on the time scale of the spike-wave complex, but cannot indicate whether chaos exits on a shorter time scale. 
Non-periodic brain waves characterize a non-seizure state which has little inter-channel correlation, as distinct from an epileptic seizure, when the EEG data has a large periodic component and a strong inter-channel correlation. Clearly, a transition occurs between these two brain states. We apply various time series analysis techniques to quantify brain dynamics, and specifically to characterize this transition and the occurrence of an epileptic seizure.

Robustness to noise and finite data precision are key issues because previous work (e.g., Ref. 33) found that noisy biological data are very difficult to analyze. Thus, our tools are adapted to handle noise, finite data precision, and finite dataset length. We also used other well-known linear statistics (data minimum and maximum, average, standard deviation, absolute average deviation, skewedness, and kurtosis). Fourier power spectral density (a linear measure) provided no clear indicators for this study of nonlinear brain activity and consequently was excluded from this study. See Ref. 20 for a discussion of the Fourier analysis and related results.

This paper is organized as follows. Section 2 explains the data acquisition and filtering techniques. Section 3 describes the features of specific nonlinear tools and their application to EEG data. Section 4 discusses the surrogate analysis, showing that statistically significant nonlinear structure is present during non-seizure, transition, and seizure epoches, thus justifying further nonlinear analysis of the EEG data. Section 5 provides a discussion of our results, showing long time-scale trends in the nonlinear features for seizure detection and prediction in this patient. Section 6 presents the conclusions of this work. 


\section{DATA AND FILTERING}

Sixteen channels of EEG data were analyzed in the bipolar montage, as illustrated in Fig. 2.1. The data were retrieved in analog form from VHS tapes and converted to digital form with 12-bit precision, giving a positive or negative, 4-digit number. The digital sampling rate $\left(f_{\mathrm{s}}\right)$ was $512 \mathrm{~Hz}$ over a total sample time of 10-23 minutes, corresponding to a total dataset size of 9.8-22.5 megabytes in binary form. Three epochs of data were examined: epileptic seizure and postseizure, non-seizure, and transition from non-seizure state to seizure (transition).

EEG data contains not only signals associated with brain activity, but also has artifacts (e.g., eye blinks, muscle twitches, chewing, etc.) that obscure the brain-wave signal. We developed a novel zero-phase filter to remove low-frequency artifacts, based on the following criterion. We needed a zero-phase-shift filter to prevent phase distortions when subtracting the filter output (the "artifact" signal) from the EEG signal to yield an undistorted artifact-filtered signal, because phase relationships are important in the subsequent nonlinear analysis. Standard high-pass filter techniques do not meet this criterion. A computationally fast, simple, low-frequency signal follower was necessary, so we eventually can apply the filter in real- or near-real time. Consequently, we used quadratic regression analysis, with the same number of data samples on either side of a central point. Other standard digital filtering methods (e.g., see Ref. 45) could not meet this requirements.

The zero-phase filter method is as follows. Ref. 20 provides a more detailed discussion of the method. For a specific EEG channel, the signal (e) at time $(t)$ is sampled at regular intervals $\left(t_{i}=i\right.$ $\Delta t$ ) to yield a set of time serial data $e_{i}=e\left(t_{i}\right)$. We choose a filter-window length of $2 n+1$ points from the time series, where $n$ is the number of points on either side of the central point $\left(e_{c}\right)$ as indicated in the below sequence.

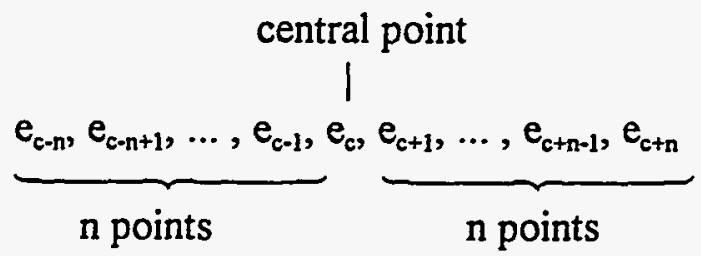

We fit the data to a quadratic equation that takes the form: $F_{i}=F\left(t_{i}\right)=a_{1}\left(t_{i}-t_{c}\right)^{2}+a_{2}\left(t_{i}-t_{c}\right)+a_{3}$ $=a_{1} T_{i}^{2}+a_{2} T_{i}+a_{3}$. Here, $t_{c}=c \Delta t$ is the time at the central point, and $T_{i}=t_{i}-t_{c}$. This approximation is fitted to the data, by minimizing the sum of squares of the differences between the quadratic equation, $F(t)$, and the raw EEG data, $e(t)$, corresponding to the minimum in the following function:

$$
L=\sum_{i=c-n}^{c+n}\left[F\left(t_{i}\right)-e\left(t_{i}\right)\right]^{2}=\sum_{i=-n}^{n}\left[\left(a_{1} T_{i}^{2}+a_{2} T_{i}+a_{3}\right)-e_{i+c}\right]^{2} .
$$




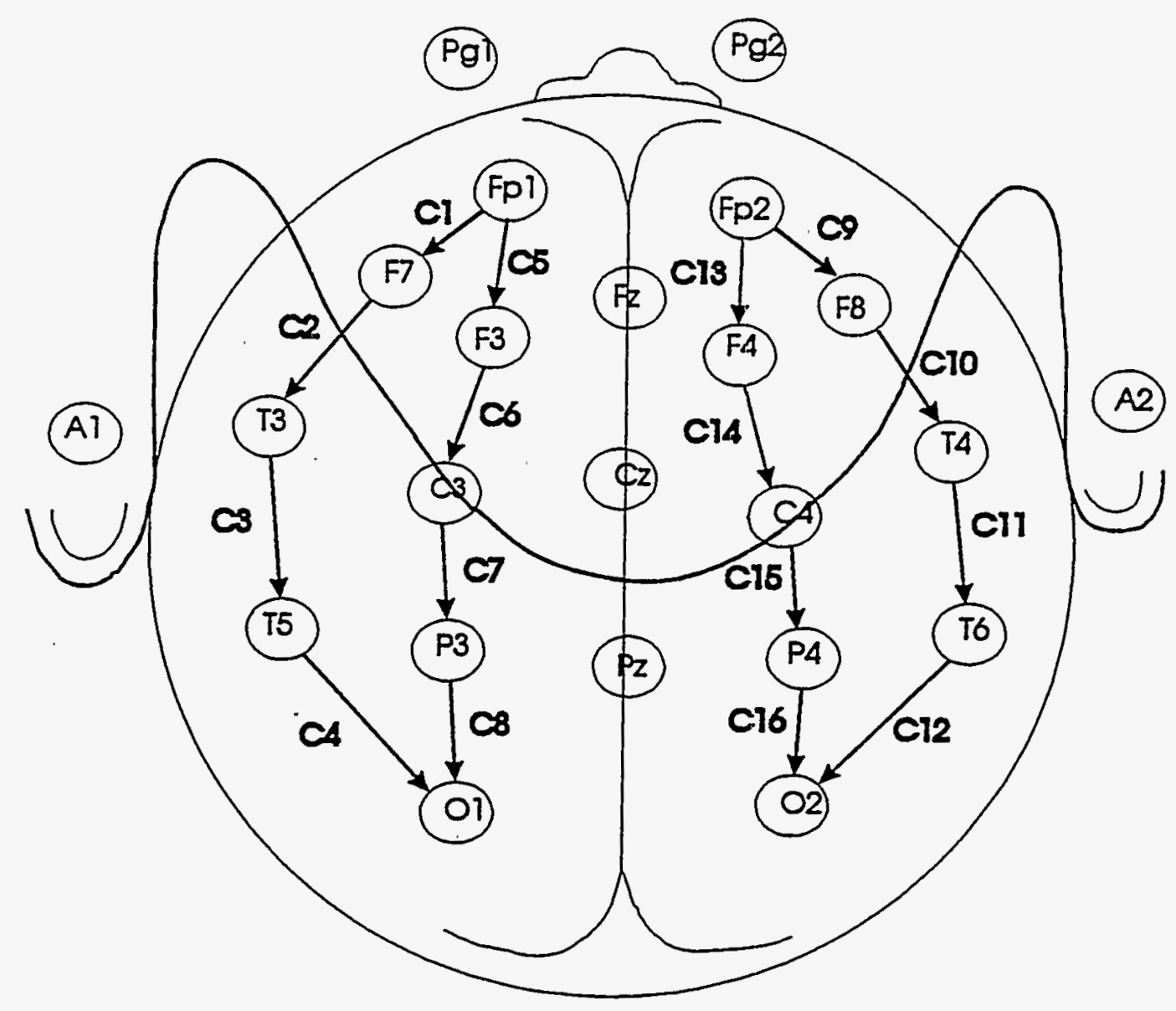

Fig. 2.1. EEG electrode positions on the patient's scalp for the bipolar montage, looking from above. C13 labels the position of the channel 13 electrode, used in this work. 
The minimum in $L$ is found from the condition $\partial L / \partial a_{k}=0$, for $k=\{1,2,3\}$, forming three simultaneous linear equations in three unknowns. The window-averaged artifact $\left(F_{c}\right)$ is given by the fitted value of the central point, $F_{c}=F(0)=a_{3}$. We note that the sums over odd powers of $T_{i}$ are zero and that symmetric sums over even powers of $T_{i}$ (over $i$ from $-n$ to $+n$ ) can be converted to sums from 1 to $n$ with $T_{i}=i \Delta t$, yielding a window-averaged solution for the artifact signal:

$$
F_{c}=\frac{3\left(3 n^{2}+3 n-1\right)\left(\Sigma_{i} e_{i+c}\right)-15\left(\Sigma_{i} i^{2} e_{i+c}\right)}{\left(4 n^{2}+4 n-3\right)(2 n+1)}
$$

Here, $\Sigma_{i}$ indicates the sum over $i$ from $-n$ to $+n$. Sums over even powers of " $i$ " were explicitly evaluated with standard formulae (e.g., see Ref. 36). The effort to evaluate $F_{c}$ can be reduced substantially by computing the sums initially from Eq. $2.2($ at $c=n+1)$, and then using the following recursions thereafter:

$$
\begin{aligned}
& \sum_{i=-n}^{n} e_{i+c+1}=e_{c+n+1}-e_{c-n}+\sum_{i=-n}^{n} e_{i+c} \\
& \sum_{i=-n}^{n} i e_{i+c+1}=n e_{c+n+1}+(n+1) e_{c-n}+\sum_{i=-n}^{n} i e_{i+c}-\sum_{i=-n}^{n} e_{i+c} \\
& \sum_{i=-n}^{n} i^{2} e_{i+c+1}=n^{2} e_{c+n+1}-(n+1)^{2} e_{c-n}+\sum_{i=-n}^{n} i_{i+c}^{2}-2 \sum_{i=-n}^{n} i e_{i+c}+\sum_{i=-n}^{n} e_{i+c} .
\end{aligned}
$$

The right-hand sides of Eqs. 2.3-2.5 only involve the sums previously computed. Application of Eqs. 2.2-2.5 to the N-point set of original time serial EEG data $\left(e_{i}\right)$ yields an artifact dataset $\left(f_{i}\right.$ or $f$-data) with $(\mathrm{N}-2 \mathrm{n})$ points that contains the low frequency artifact signal. The residual signal ( $g_{\mathrm{i}}$ or g-data) is the difference, $g_{i}=e_{i}-f_{i}$, and is a signal that as free of low-frequency artifacts. Subsequently, we apply a standard fourth-order low-pass filter at $50 \mathrm{~Hz}$ (e.g., see Ref. 32) to the g-data, to yield artifact-filtered, low-pass-filtered data $\left(h_{i}\right.$ or $h$-data) that is free of both low- and high-frequency artifacts. We note that spike-wave phenomena at $100 \mathrm{~Hz}$ in h-data are attenuated by $28 \mathrm{db}$ (a factor of 25), while the g-data retain the full spike-wave signals.

The filter-window length ( $n=128)$ corresponds to a frequency of $2.0 \mathrm{~Hz}[=512 \mathrm{~Hz} /(2 \mathrm{n}+1)]$. Figure 2.2a shows an example of the application of this method, with (raw) e-data in light gray and a superimposed (dark line) artifact signal (f-data), which clearly follows the low-frequency trends. Figure 2.2b shows the residual signal (g-data) for this example, as having little lowfrequency component while retaining the higher frequency information.

For a specific EEG channel, we obtained a time history of the nonlinear measures by applying the CTSA tools to a series of 20-second analysis-windows of the four data types (e, $f, g, h$ ). These data are designated in Section 3 as $x_{i}$. The length of the analysis window (w) was 10,240 points. Each analysis-window had a 5,120-point overlap with the previous (or next) analysis- 

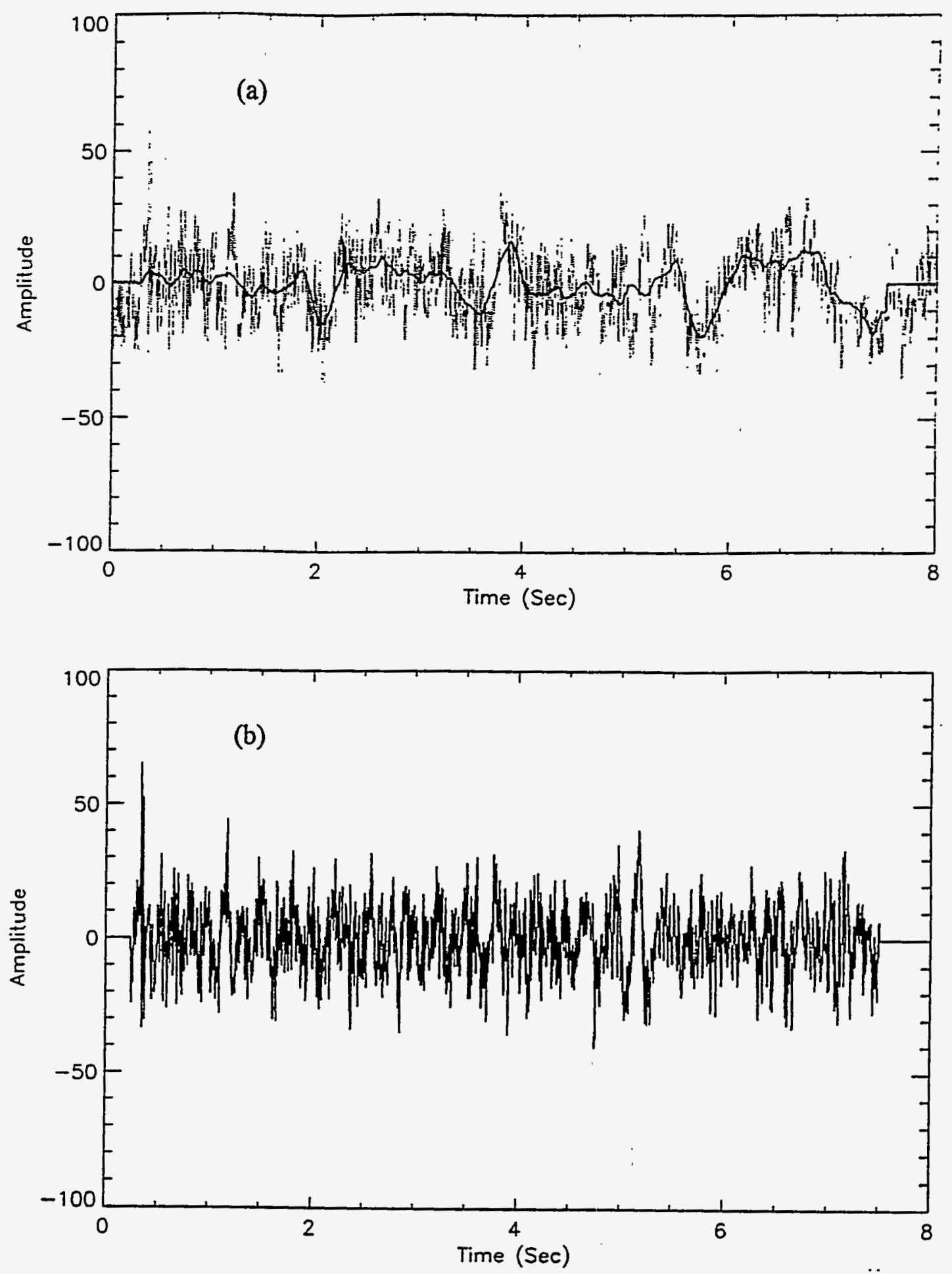

Fig. 2.2. Sample plots of non-seizure EEG data. In the upper plot (a), a short sample of raw EEG (e-) data from the non-seizure part of dataset \$73317 (rapidly varying, gray points) and corresponding artifact (f-) data (dark solid line) from the zero-phase quadratic filter. The lower plot $(b)$ shows the artifact-filtered $(g-)$ data, by subtracting the f-data from the e-data for this sample data segment. 
window of data. This $50 \%$ overlap provides an optimal mix of new and old data for smooth timehistory trend generation [11], as illustrated below.

$\mid<---0$ to 20 seconds--- $>\mid$

$<---10$ to 30 seconds---->1

$\mid<---20$ to 40 seconds---- $\mid$

$<--100$ to 120 seconds-- $>\mid$

$\mid<-110$ to 130 seconds-- $>\mid$

The zero-phase quadratic filter provides artifact-filtered data with frequencies of $\geq 2 \mathrm{~Hz}$. A heuristic for (linear) Fourier analysis is that $\geq 10$ periods of data are required to faithfully recover cyclic information at a specific frequency. Thus, $\geq 5$ seconds of data are needed to obtain Fourier amplitude and phase information at a signal frequency of $2 \mathrm{~Hz}$. However, this heuristic does not apply to nonlinear analysis. For example, 20 seconds of data are necessary to obtain consistent results for the Kolmogorov entropy. This need for longer dataset lengths ( $\sim 10,000$ points) for consistent nonlinear measures conflicts with the need for shorter dataset lengths $(\leq 5,000$ points) to provide adequate resolution for the time history generation of trends. Consequently, we used a 20 -second analysis window, as described above, with a $50 \%$ overlap for an effective time history resolution of 10 seconds. The nonlinear measures for each 20 -second analysis window were associated with the time at the center of the analysis window, i.e., every ten seconds. 


\section{NONLINEAR ANALYSIS TOOLS FOR EEG DATA}

Many characterization tools exist for chaotic data analysis. Due to very limited resources, we used a subset of the tools that were found by previous work to be good measures for EEG data, as discussed in Section 1. These tools include the following:

- standard statistical measures (minimum, maximum, average, absolute average deviation, standard deviation, skewedness, kurtosis, time per cycle);

- Kolmogorov entropy and entropy spectrum;

- mutual information function;

- maximum likelihood correlation dimension and correlation dimension spectrum;

- surrogate generation and nonlinearity tests;

- nonlinear digital filters (as discussed in Section 2).

We justify the choice of these nonlinear analysis tools as follows. Weigend and Gershenfeld [51] report a relationship [10,27,43] between epileptic seizures and the correlation dimension of EEG data. As discussed in Section 1, two other measures, entropy and mutual information, also show a relationship $[10,17,27]$ to a seizure. The first minimum in the mutual information defines the time scale for generating the return map for EEG dynamics. The return map underlies the correlation dimension (measure of dynamic complexity) and entropy (measure of dynamic predictability). Moreover, we have had good success with these same measures in analyzing other systems. Consequently, entropy, correlation dimension, and mutual information were used in the present study as nonlinear measures for seizure analysis.

The statistical measures for the present study are obtained by standard methods (e.g., Ref. 18). The maximum and minimum are obtained as maximum and minimum (respectively) over the $x_{i}$ values in a time-serial window of $\mathrm{w}$ points. The average $(\underline{\mathrm{x}})$ is given by:

$$
\underline{x}=(1 / w) \sum_{i=1}^{w} x_{i}
$$

The $r$-th order moment $\left(m_{r}\right)$ of the $x$-data is:

$$
\mathrm{m}_{\mathrm{T}}=(1 / \mathrm{w}) \sum_{i=1}^{\mathrm{w}}\left(\mathrm{x}_{i}-\underline{\mathrm{x}}\right)^{\mathrm{r}} \text {. }
$$

The absolute average deviation (a) provides a robust indicator of the $x_{i}$ variability $[29,40]$ and is defined as:

$$
a=(1 / w) \sum_{i=1}^{w}\left|x_{i}-\underline{x}\right| \text {. }
$$

An unbiased estimate of the standard deviation $(\sigma)$ is: 


$$
\sigma=\left[\mathrm{w} \mathrm{m}_{2} /(\mathrm{w}-1)\right]^{1 / 2} .
$$

An estimate for the skewedness (s) is:

$$
\mathrm{s}=\mathrm{m}_{3} / \mathrm{m}_{2}^{3 / 2} .
$$

An estimate for the kurtosis $(\mathrm{k})$ is:

$$
\mathrm{k}=\mathrm{m}_{4} / \mathrm{m}_{2}^{2}-3 \text {. }
$$

The average cycle time $\left(T_{c}\right)$ is important as a characteristic time of the nonlinear system:

$$
T_{c}=\text { (window length in timesteps) } /[\text { (number of mean crossings) } / 2]=2 \mathrm{w} / \mathrm{c} \text {. }
$$

The mutual information function (MIF) is a nonlinear version of the (linear) auto-correlation and cross-correlation functions, and was originally developed by Shannon and Weaver [41] with subsequent application to time series analysis by Fraser and Swinney [13]. Mutual information measures the certainty with which a measurement can be predicted, given the outcome of another related measurement. Examples of the later include the same EEG channel at a different time, and another EEG channel at the same (or different) time. The MIF indicates the average information (in bits) that can be inferred from one measurement about a second measurement, and is a function of the time delay (number of time steps) between the measurements. The mutual information function also measures the nonlinear time dependent correlation in the same signal. For EEG data, the MIF is useful for three reasons. First, information decay in an individual channel (univariate MIF) indicates local time scale, as the average time lag $\left(t_{j}-t_{j}\right)$ that makes $x\left(t_{i}\right)$ independent of $x\left(t_{j}\right)$, and corresponds to the first (local) minimum $\left(M_{1}\right.$ in timesteps) in the MIF [13]. We defined a minimum as two successive decreases in the signal value, followed by two successive increases in signal value. Other definitions were tested and found to yield less consistent results. Second, the MIF between channels (bivariate MIF) can reveal dynamic coupling of different spatial regions of the brain and the propagation time between them. Third, the uni- and bi-variate MIF measures timescale changes in local/global dynamics from non-seizure state, through transition, to a seizure. The scope of the present work did not permit a study of the second and third aspects, which are reported elsewhere [20]. The MIF, I(Q,S), and system entropy $(\mathrm{H})$ for two measurements ( $\mathrm{Q}$ and $\mathrm{S}$ ) are defined by:

$$
\begin{aligned}
& I(Q, S)=I(S, Q)=H(Q)+H(S)-H(S, Q), \\
& H(S)=-\sum_{i} P_{S}\left(s_{i}\right) \log \left[P_{S}\left(s_{i}\right)\right] \\
& H(S, Q)=-\sum_{i, j} P_{S Q}\left(s_{i}, q_{j}\right) \log \left[P_{S Q}\left(s_{i}, q_{j}\right)\right] .
\end{aligned}
$$

$\mathrm{S}$ denotes the whole system that consists of a set of possible messages (measurements for the value of $s), s_{1}, s_{2}, \ldots, s_{n}$ with associated probabilities $P_{s}\left(s_{1}\right), P_{s}\left(s_{2}\right), \ldots, P_{s}\left(s_{n}\right)$. $Q$ denotes a 
second system that consists of a set of possible messages (measured values with a time delay relative to the $s_{i}$ values), $q_{1}, q_{2}, \ldots, q_{n}$ with associated probabilities $P_{Q}\left(q_{3}\right), P_{Q}\left(q_{2}\right), \ldots, P_{Q}\left(q_{n}\right)$. The function $P_{S Q}\left(s_{i}, q_{j}\right)$ denotes the joint probability of both states occurring simultaneousiy. If the logarithm is taken to the base two, then $\mathrm{H}$ is in units of bits. Fraser and Swinney [13] describe the details for evaluating $I(Q, S)$, including a sequence of recursive partitions in $\left(s_{i}, q_{j}\right)$ space to achieve adequate accuracy with tailoring to the local data structure.

The maximum-likelihood correlation dimension (D) is based on the early work by Takens [44] with modifications for noise [40]:

$$
D=\left[(-1 / M) \sum_{i, j} \ln \left[\left(r_{i j}-r_{n}\right) /\left(1-r_{n}\right)\right]\right]^{-1},
$$

where $M$ is the number of randomly sampled point pairs, $r_{i j}$ is the normalized maximum-norm distance between the (randomly chosen) $\mathrm{i}$-j point pairs as defined in Eq. 3.12 (below), and $r_{n}$ is the normalized distance (scale length) associated with noise as measured from the time serial data. The distances are normalized with respect to some nominal scale length $\left(L_{0}\right)$, i.e. $r_{i j}=L_{i j} / L_{0}$ and $r_{n}$ $=\mathrm{L}_{\mathrm{n}} / \mathrm{L}_{0}$ with $\mathrm{L}_{0}$ as a representative scale length (typically a multiple of the absolute average deviation). The choice of scale length is a balance between a small scale for sensitivity to local dynamics (typically at $\mathrm{L}_{0}<5 \mathrm{a}$ ) and avoidance of excessive noise (typically at $\mathrm{L}_{0} \geq \mathrm{a}$ ). The distances $\left(L_{i j}\right)$ are defined by:

$$
L_{i j}=\max _{0 \leq k \leq m-1}\left|x_{i+k}-x_{j+k}\right|
$$

where $m$ is the average number of points per cycle from Eq. 3.7 (i.e., $m=T_{c}$ ). Schouten et al. [40] describe the details for evaluating Eqs 3.11-3.12 to measure of the number of degrees of freedom in a system (e.g., the number of coupled first-order differential equations to depict the dynamics).

The Kolmogorov entropy (K-entropy or simply entropy) measures the rate of information loss per unit time, or (alternatively) the degree of predictability. A positive, finite entropy generally is considered to be a clear demonstration that the time series and its underlying dynamics are chaotic. An infinite entropy indicates a stochastic, non-deterministic (totally unpredictable) phenomenon. For entropy determination, one begins with two orbits on a chaotic attractor that are initially very close together. The entropy then is estimated from the average divergence time for pairs of initially-close orbits. More precisely, the entropy is obtained from the average time for two points on an attractor to go from an initial separation $\left(L<L_{0}\right)$, to become separated by more than a specific distance $\left(\mathrm{L} \geq \mathrm{L}_{0}\right)$. The maximum-likelihood entropy is obtained from the method by Schouten, Takens, and van den Bleek [39]:

$$
\begin{aligned}
& K=-f_{s} \log (1-1 / \underline{b}), \text { and } \\
& \underline{b}=(1 / M) \sum_{i=1}^{M} b_{i},
\end{aligned}
$$


with $b_{i}$ as the number of timesteps for two points, initially within $L<L_{0}$, to diverge to $L \geq L_{0}$. The work by Schouten et al. [39], and references therein, provide details of the method. We note that the entropy used here is the order-2 Kolmogorov entropy which hereafter we call simply entropy.

The entropy $(\mathrm{K})$ and correlation dimension (D) usually are reported in the limit of zero scale length. However, EEG data (and all biomedical data) have substantial noise. Consequently, we report the nonlinear measures of $\mathrm{K}$ and $\mathrm{D}$ for a finite scale length $\left(\mathrm{L}_{0}\right)$ that is slightly larger than the noise. Thus, the values of $K$ and $D$, that we report here, do not capture the full complexity of brain dynamics, i.e., their values are smaller than expected for the zero-scale-length limit. We interpret $\mathrm{K}$ and $\mathrm{D}$ as nonlinear statistical indices of finite-scale dynamic structure.

We used surrogate analysis to show that significant nonlinear structure is present in the data. Previous work [34] showed that random-phase surrogate analysis can give a dramatically, and totally spurious, identification of non-random structure in dynamical data. Thus, we follow the recent examples of surrogate analysis $[30,34]$, which use both phase randomization and data shuffling. The methodology begins by applying a discrete Fourier transform $(\mathcal{I})$ to the e-data:

$$
Z(f)=\mathcal{J}\{e(t)\}=\sum_{n=1}^{N-1} e\left(t_{n}\right) e^{2 \pi j j n \Delta t}
$$

where $e_{i}=e\left(t_{i}\right)$ is the raw EEG data as discussed previously, and $Z(f)$ is a set of complex Fourier amplitudes, $\left\{X_{\mathrm{k}}+\mathrm{j} \mathrm{Y}_{\mathrm{k}}\right\}$, for the discrete frequencies $\mathrm{f}=-\mathrm{N} \Delta \mathrm{f} / 2, \ldots,-\Delta \mathrm{f}, 0, \Delta \mathrm{f}, \ldots, \mathrm{N} \Delta \mathrm{f} / 2$, with $\Delta f=1 / N \Delta t$. We note that " $j "$ in this context indicates the square root of -1 . Then, a phaserandomized Fourier transform, W(f), results from rotating the phase at each frequency by an independent random variable $(\phi)$, in the range $0 \leq \phi \leq 2 \pi$ :

$$
W(f)=Z(f) e^{j \phi}=\left(X_{k}+j Y_{k}\right)(\cos \phi+j \sin \phi)=\underset{j\left(Y_{k} \cos \phi+X_{k} \sin \phi\right) .}{X_{k} \cos \phi-Y_{k} \sin \phi+}
$$

We note that proper reconstruction of the phase-randomized time series requires that the Fourier component for the negative frequency be the complement of that for the positive frequency, i.e., $W(-f)=W^{*}(f)$. A phase-randomized time series, $p_{i}=p(t)$, next is constructed by applying the inverse discrete Fourier transform $\left(\mathcal{f}^{-1}\right)$ to $W(f)$ :

$$
p(t)=g^{-1}[W(f)]=\sum_{n=1}^{N-1} W\left(f_{n}\right) e^{-2 \pi j f n \Delta t} .
$$

By construction, $p(t)$ has the same power spectrum as the original data. Moreover, $p(t)$ and the original data have the same circular autocorrelation function, $A(\tau)$ :

$$
A(\tau)=\sum_{n=1}^{N-\tau}\left[x\left(t_{n}\right)-\underline{x}\right]\left[x\left(t_{n+\tau}\right)-\underline{x}\right] / N+\sum_{n=N-\tau+1}^{N}\left[x\left(t_{n}\right)-\underline{x}\right]\left[x\left(t_{n+\tau-N}\right)-\underline{x}\right] / N .
$$


Subsequently, both the original data and the $p(t)$ data are rank-ordered from smallest to largest value, i.e. $p_{R 1} \leq p_{R 2} \leq \ldots \leq p_{R N}$ and $x_{R 1} \leq x_{R 2} \leq \ldots \leq x_{R N}$, while retaining the correspondence between the time serial index and the rank order. We obtain the surrogate data $\left(\mathrm{q}_{\mathrm{i}}\right)$ by replacing the phase-randomized data $\left(\mathrm{p}_{\mathrm{i}}\right)$ of rank $(\mathrm{R})$ with original data $\left(\mathrm{e}_{\mathrm{i}}\right)$ of equal rank $(\mathrm{R})$. This procedure is called data shuffling. Both $e_{\mathrm{i}}$ and the surrogate dataset have the same Fourier spectrum, the same autocorrelation function, and the same probability density function. Finally, we applied the nonlinear analysis tools to $\mathrm{q}_{\mathrm{i}}$, with the same cutoff scale length $\left(\mathrm{L}_{0}\right)$ as described earlier in this Section. 


\section{SURROGATE ANALYSIS}

We used the surrogate methodology on an EEG dataset to obtain 20 surrogate datasets for a 200 -second analysis window (102,400 points) in the middle of the non-seizure epoch (100-300s). The difference $(\Delta)$ between the real and surrogate measures (denoted by superscripts " $\mathrm{r}$ " and "s," respectively) indicates the degree of nonlinearity, $\Delta_{V}=\left(V^{T}-V^{v}\right) / \sigma_{v}$, with " $V^{\prime}$ refering generically to each nonlinear measure. The surrogate averages and standard deviations (denoted by $\mathrm{V}^{3}$ and $\sigma_{\mathrm{v}}$, respectively) were computed from the 20 surrogate estimates of the nonlinear measures, using Eqs. 3.1 and 3.4. We repeated the surrogate analysis for a 200-second analysis window (102,400 points) in the middle of the transition epoch (700-900s) and for the 180-second seizure epoch (1200-1380s). Long datasets provide a strong statistical basis for the comparisons, but use of a 10,240-point analysis window shows much variability (e.g., Sect. 5). Table 4.1 shows the results.

Table 4.1 Surrogate results for dataset $\# 73305$

\begin{tabular}{|c|c|c|c|c|}
\hline Measure & $\mathrm{T}_{\mathrm{c}}$ & $\mathrm{K}$ & $\mathrm{M}_{1}$ & $\mathrm{D}$ \\
\hline \multicolumn{5}{|c|}{ Non-seizure } \\
\hline $\mathrm{V}^{\mathrm{r}}$ & 20.7 & 0.0145 & 91 & 1.68 \\
\hline$V^{s}$ & 13.4 & 0.0530 & 4 & 2.54 \\
\hline$\sigma_{\mathrm{v}}$ & 0.13 & 0.0059 & 0 & 0.12 \\
\hline$\Delta_{\mathrm{V}}$ & 56 & -6.5 & $\infty$ & -7.2 \\
\hline \multicolumn{5}{|c|}{ Transition } \\
\hline$V^{r}$ & 21.9 & 0.0059 & 99 & 1.23 \\
\hline$V^{s}$ & 26.5 & 0.0128 & 114 & 1.42 \\
\hline$\sigma_{\mathrm{v}}$ & 0.39 & 0.0019 & 12 & 0.10 \\
\hline$\Delta_{\mathrm{V}}$ & -11.8 & -3.6 & -1.3 & -1.9 \\
\hline \multicolumn{5}{|l|}{ Seizure } \\
\hline $\mathrm{V}^{r}$ & 20.9 & 0.0273 & 52 & 2.23 \\
\hline$V^{3}$ & 13.2 & 0.1701 & 4 & 3.91 \\
\hline$\sigma_{\mathrm{v}}$ & 0.11 & 0.0082 & 0 & 0.22 \\
\hline$\Delta_{\mathrm{V}}$ & 70 & -17.4 & $\infty$ & -7.6 \\
\hline
\end{tabular}

A large value of $\left|\Delta_{\mathrm{v}}\right|$ indicates that the original data is nonlinear, but the choice of "large" is arbitrary for $\left|\Delta_{\mathrm{v}}\right| \geq 3$ (i.e., $23 \mathrm{SDs}$ ), because the probability for such outliers is small. For example, the Gaussian probabilities are $1.4 \times 10^{-3}, 9.9 \times 10^{-10}$, and $7.6 \times 10^{-24}$ for random differences $\left(\Delta_{\mathrm{V}}\right)$ of 23,26 , and $\geq 10$ SDs, respectively. The non-seizure and seizure periods show $\left|\Delta_{v}\right|>6$ for all four nonlinear measures; the transition period shows $\left|\Delta_{v}\right|>6$ for one nonlinear measure $\left(T_{c}\right)$. These results indicate that the EEG data contain significant nonlinear structure. 


\section{RESULTS AND DISCUSSION}

We analyzed two datasets, both of which are from a single patient who is 20 years of age with a lifelong history of seizures, beginning at 4 months of age. The cause of the seizures is not established, although neuro-imaging studies (including computerized tomography and magnetic resonance) are normal. The seizures are poorly controlled despite treatment with various combinations of anti-epileptic drugs, which at the time of the recordings were Phenytoin, Phenobarbital, and Felbamate. The seizures are partial complex with some occasions of secondary generalization. During the first seizure (dataset \#73305), the patient is lying in bed, awake with her right upper extremity in a flexed posture. The EEG shows spike-wave discharges in the left hemisphere, after which the brain waves become very sharp, dominated by high frequency activity and artifacts. The patient showed eye deviation to the right and some head turning to the right, with head jerking also to the right, but without any usual posturing of the right upper extremity. The eye/head turning is preceded by a high-frequency vocalization. After the clinical seizure spontaneously terminated, the EEG shows high amplitude wave slowing and subsequent amplitude suppression. The subject remained awake during the seizure but was poorly responsive. During the second seizure (dataset \#73317), the patient was sitting up in bed, doing some neurophysiologic testing. Her EEG shows an activated pattern. She then showed automatisms with picking movements and staring, followed by vocalizations (several seconds of screams). Hyper-extended head and neck posturing followed. Her upper extremities became flexed, and then she showed clonic activity, involving abduction/adduction at the shoulders and hips. There was tonic posturing and clonic activity of all extremities. The convulsive movements were associated with high-amplitude EEG waves, involving spikes, polyspikes, and much artifact activity. As the clinical seizure spontaneously terminated, the subject was unresponsive and made loud snoring sounds. Then, the brain wave amplitudes became quite suppressed. The automatisms were associated with polyspike discharges from the left frontal region. After seizure termination, spike discharges occurred from this same region, followed by suppressed background waves.

The results from dataset \#73317 involved four analyses (e-, f-, g-, and h-data) on the three epochs of channel 13 data (epileptic seizure and post-seizure, non-seizure, and transition from non-seizure to seizure). These three epochs of data were provided and analyzed as three separate, non-contiguous ten-minute data segments. The non-seizure data segment ended several hours before the transition data, which in turn ended $\sim 10$ seconds before the start of the seizure data segment. The results are combined as one thirty-minute set of plots for the e-data (Fig. 5.1), fdata (Fig. 5.2), g-data (Fig. 5.3), and h-data (Fig. 5.4), with gaps in the analysis to indicate where one dataset ends and the next dataset begins. In particular, the data from 10-590s is the nonseizure epoch with large amplitude signals at 520-600s from chewing and drinking. The data from $610-1190 \mathrm{~s}$ is the (transition) period immediately before seizure. The data from 1210-1790s includes the seizure and post-ictal phases. The various measures were obtained for every analysiswindow, and the resulting values were plotted at the center of the 20 -second analysis-window.

Each plot in Figs. 5.1 - 5.4 for dataset \#73317 displays the analysis-window-centered measure as a solid line. The dashed line (-- ) in each figure is the average value of the measure (from 

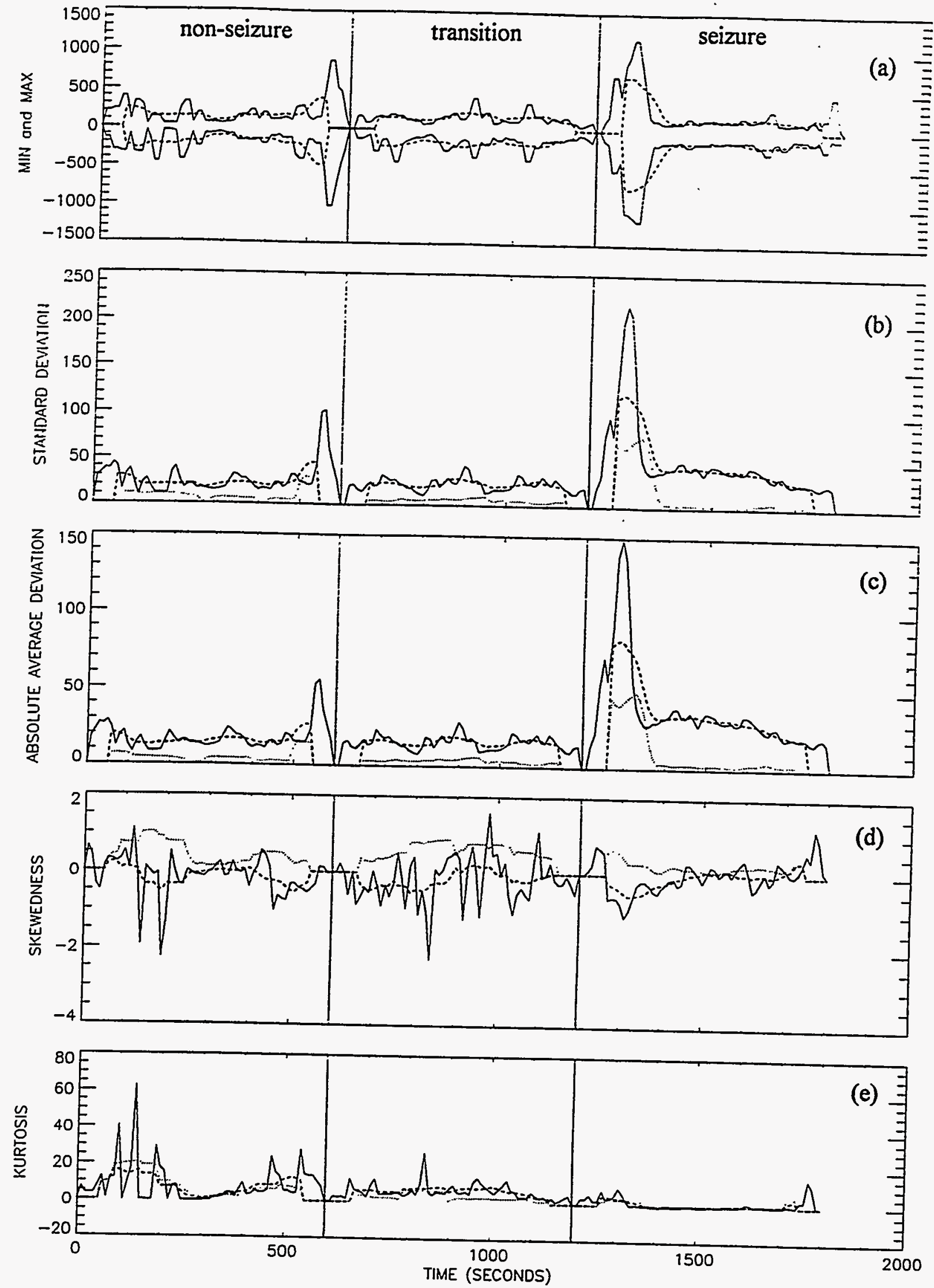

Fig. 5.1. Results for raw EEG (e-) data for dataset \#73317. The solid line is the specific measure, with the 11-point average (---) and standard deviation (...) of the measure. 

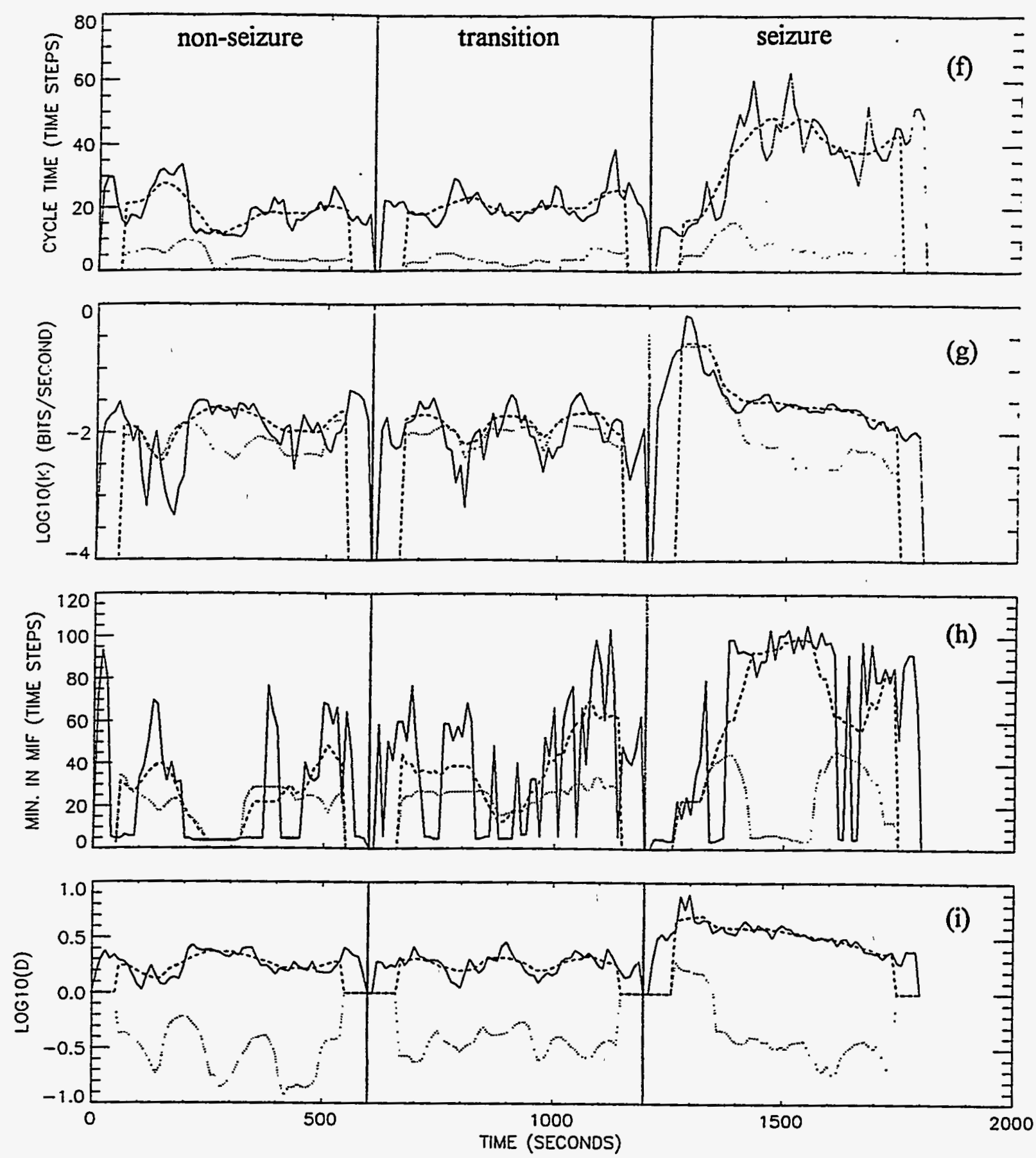

Fig. 5.1. (continued) 

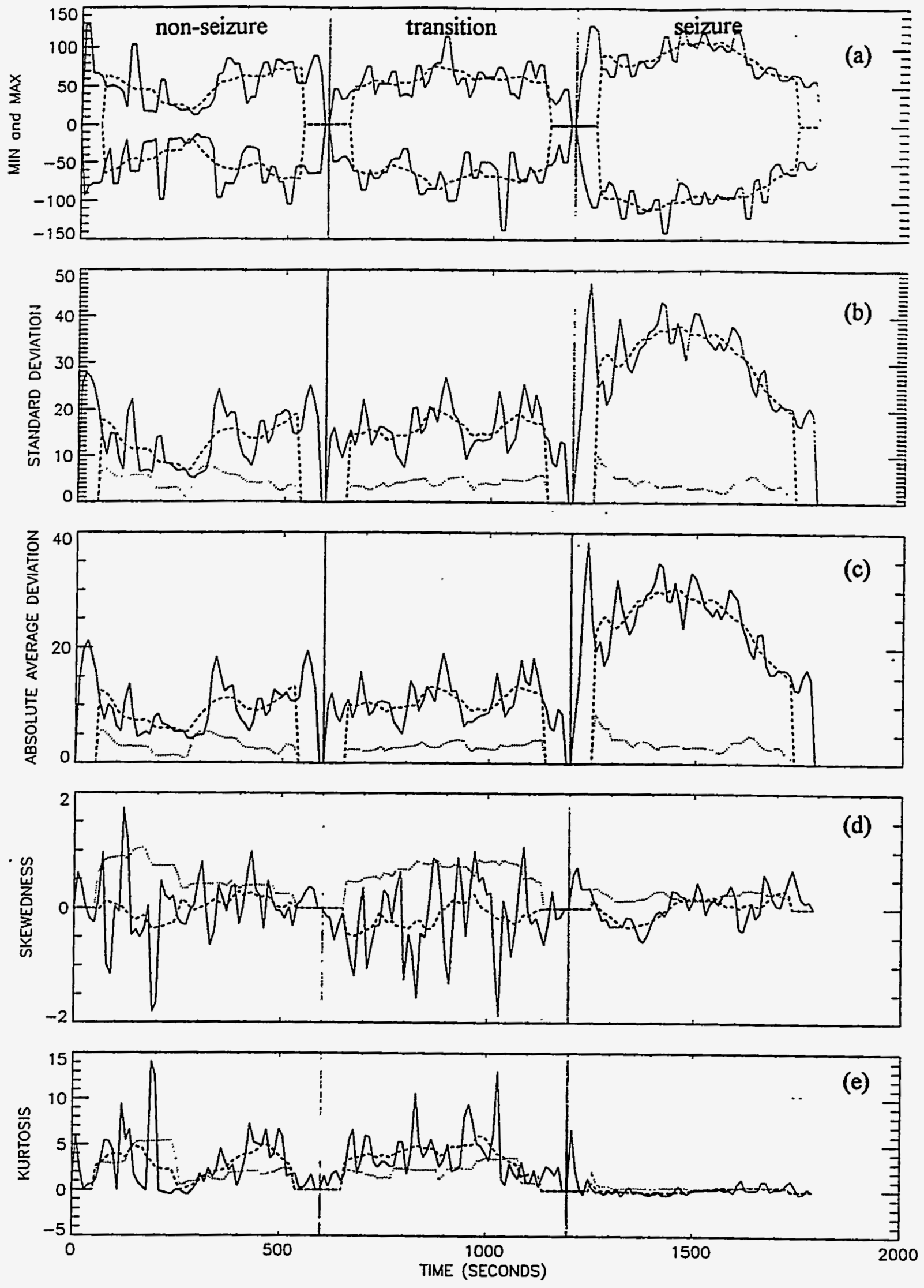

Fig. 5.2. Results for artifact EEG ( $f$ ) data for dataset \#73317. The solid line is the specific measure, with the 11-point average (--) and standard deviation (...) of the measure. 

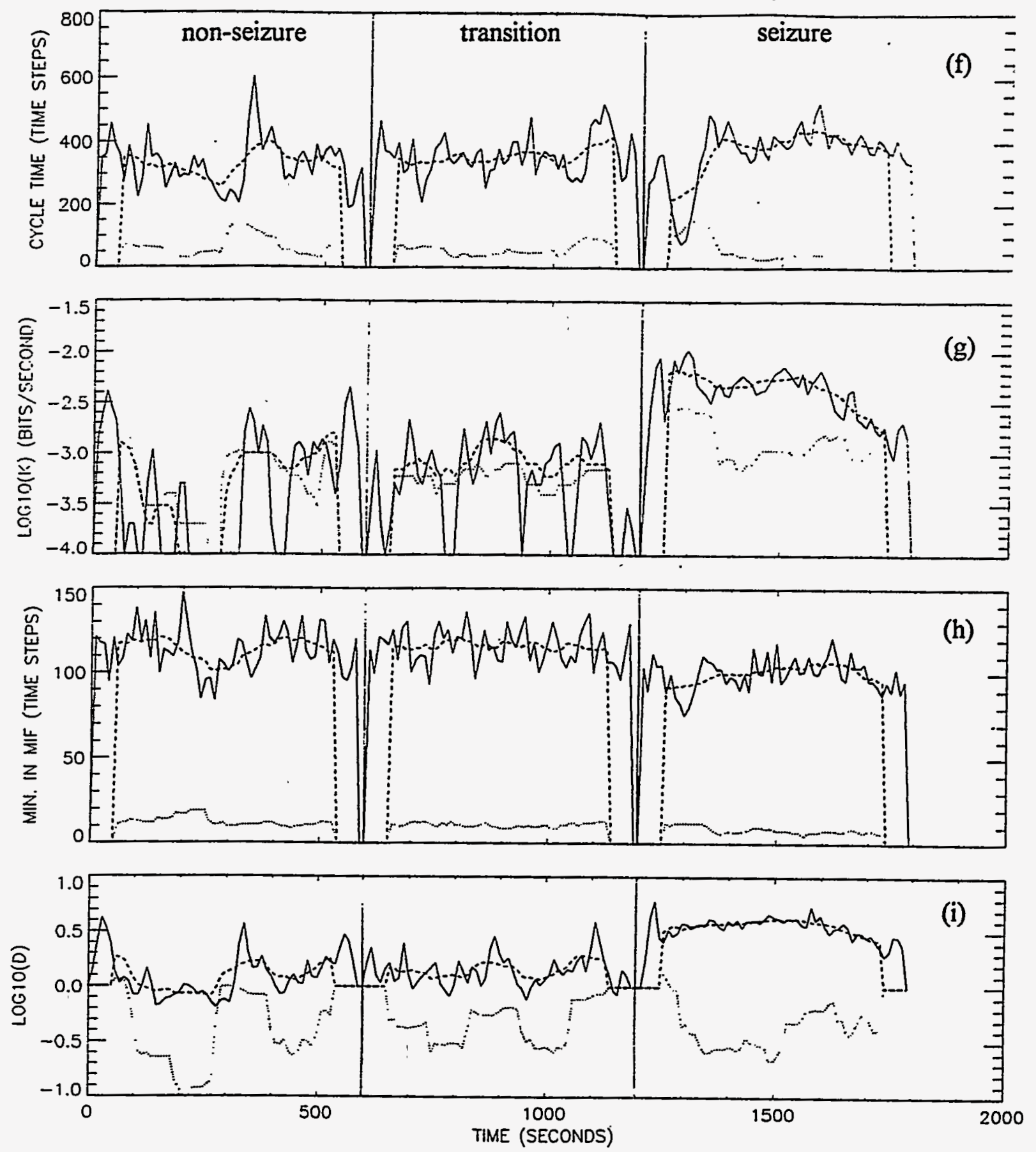

Fig. 5.2. (continued) 

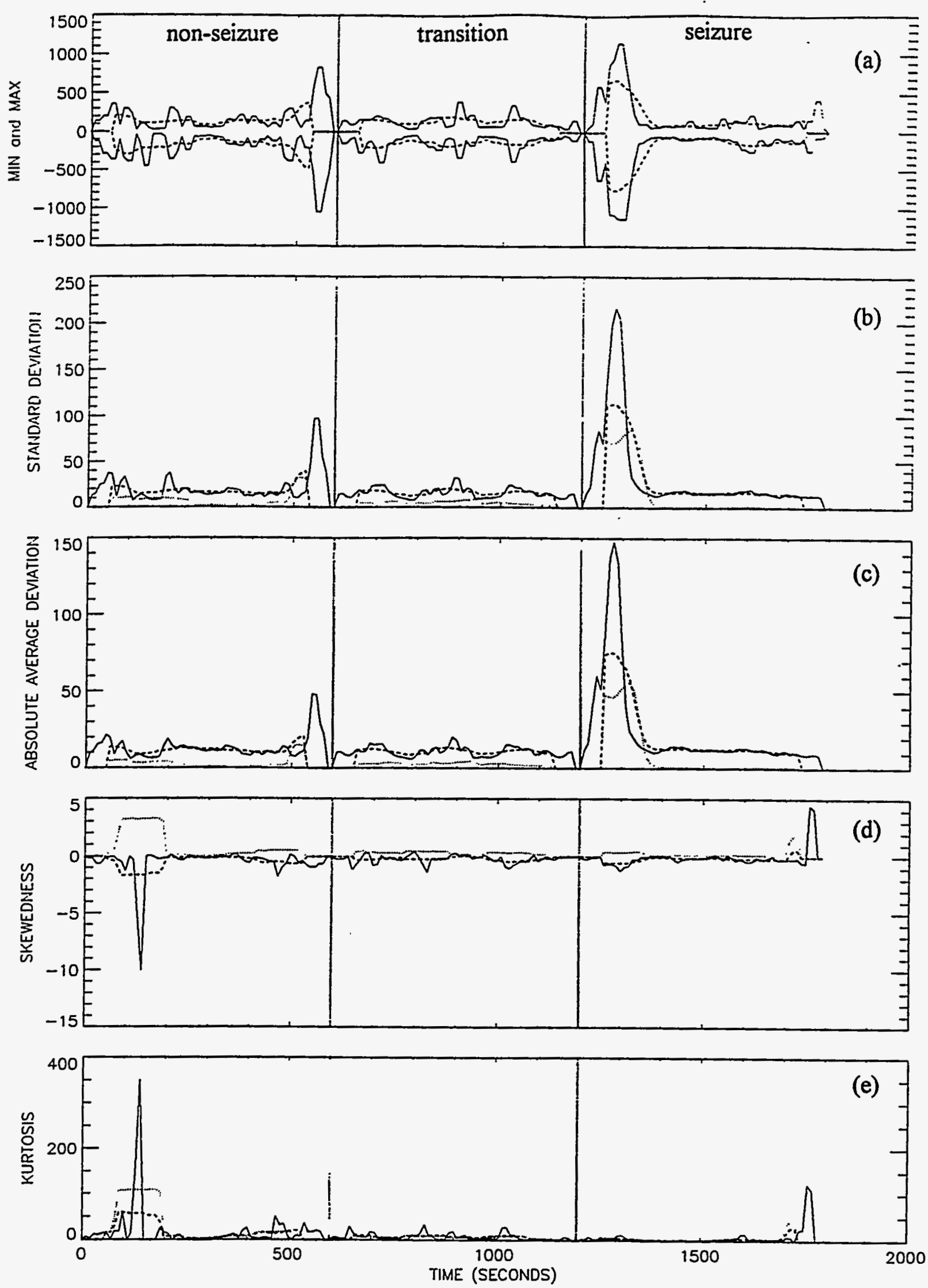

Fig. 5.3. Results for artifact-filtered EEG (g-) data for dataset \#73317. The solid line is the specific measure, with the 11-point average (--) and standard deviation (...) of the measure. 

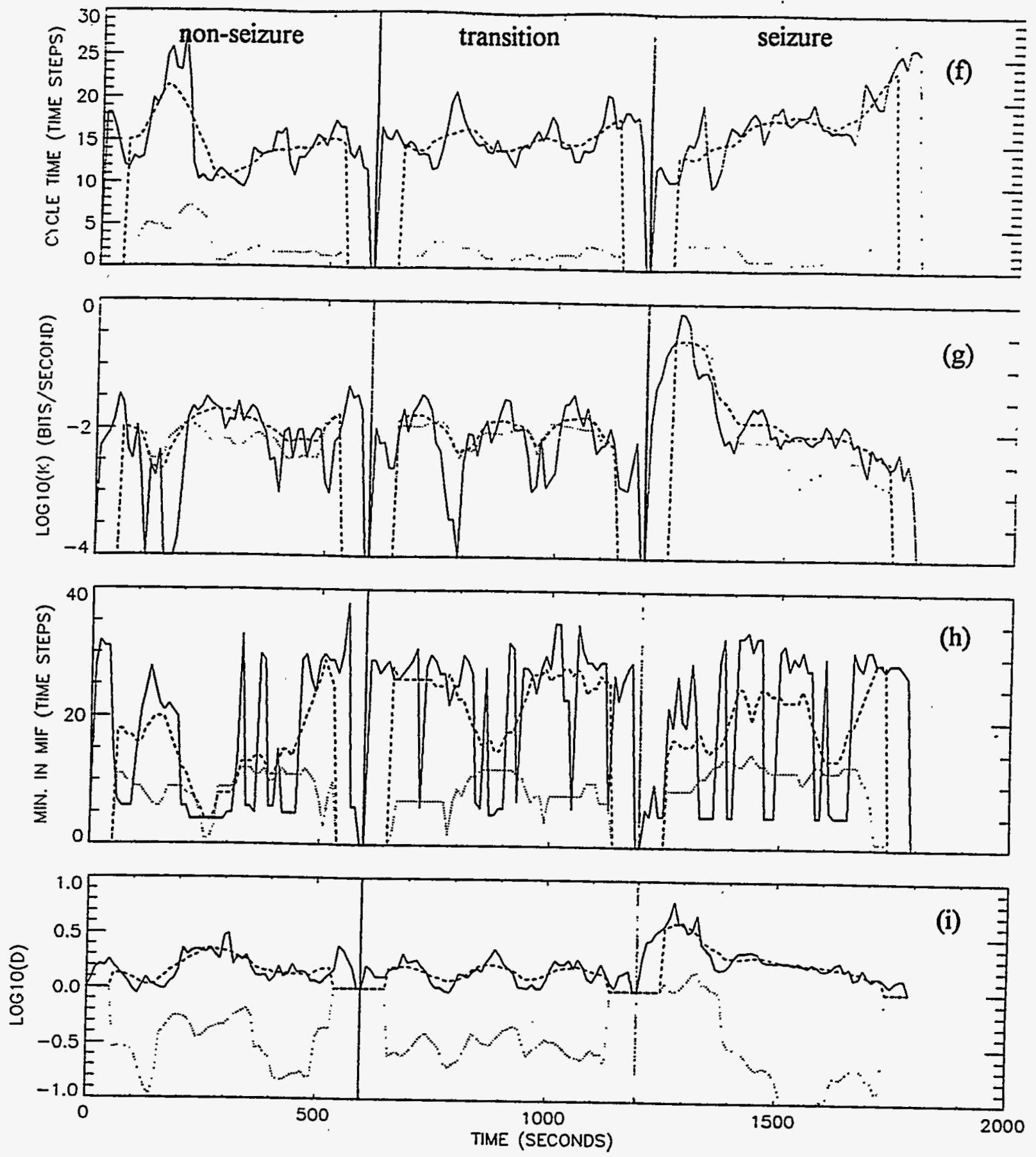

Fig. 5.3. (continued) 

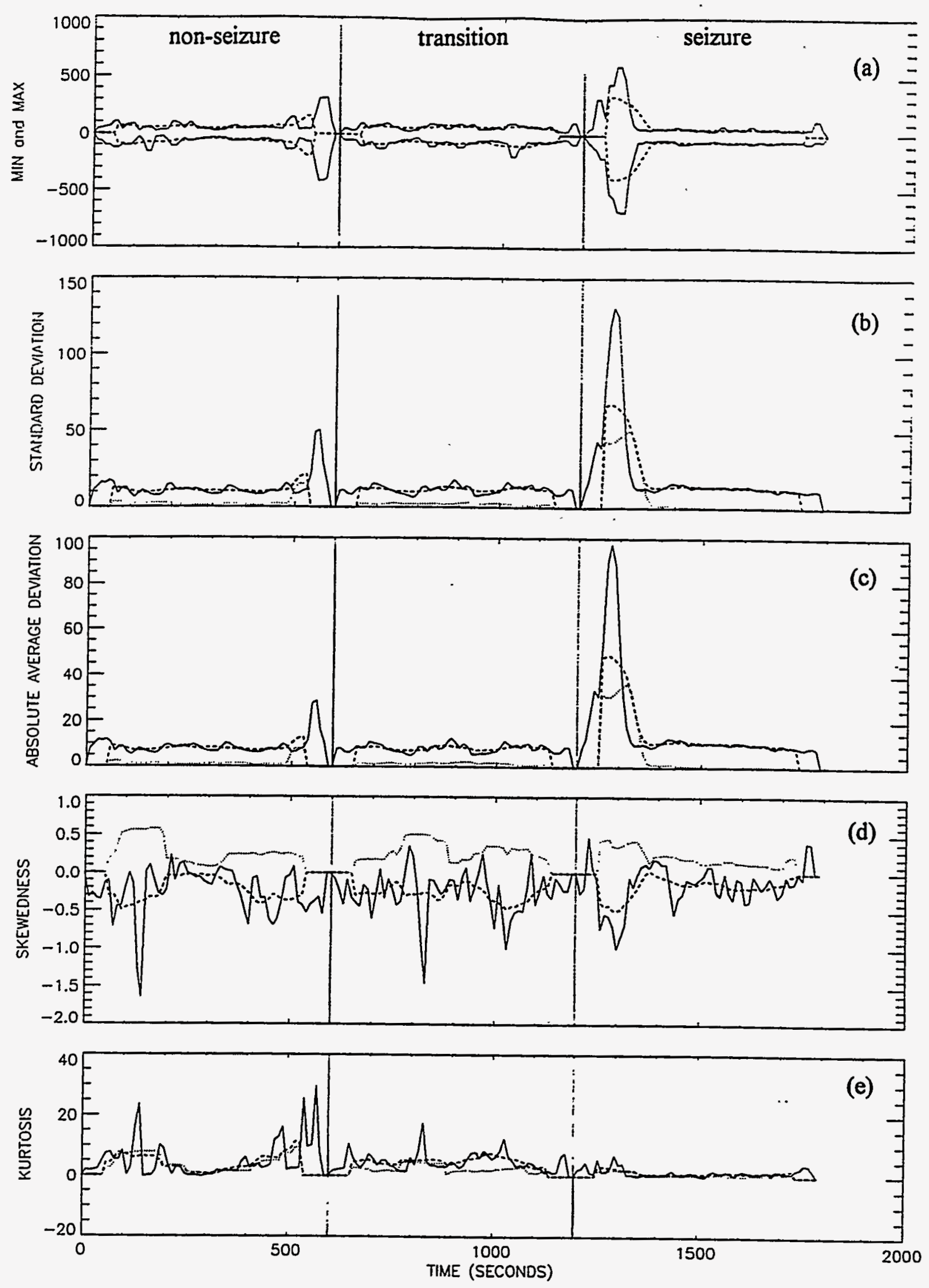

Fig. 5.4. Results for artifact- and low-pass-filtered EEG (h-) data for dataset $\# 73317$. The solid line is the specific measure, with the 11-point average $(--)$ and standard deviation (...) of the measure. 

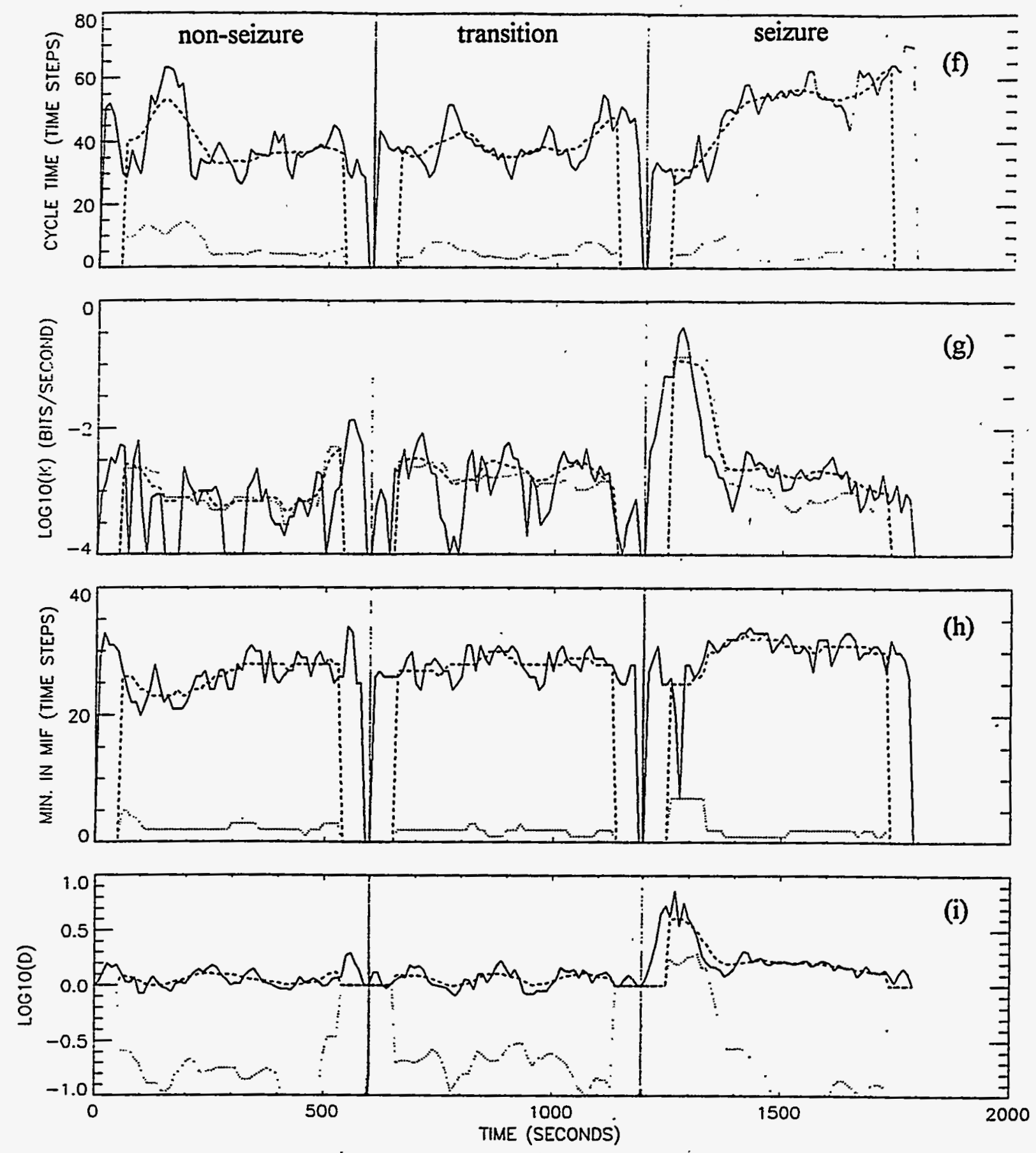

Fig. 5.4. (continued) 
Eq. 3.1) over an 11-point averaging-window, plotted at the central (sixth) point of the averaging window. The dotted line (. . ) in each figure is the corresponding sample standard deviation over this 11-point averaging-window (from Eq. 3.4), also plotted at the central point of the averagingwindow. The scale length $\left(L_{0}\right)$ was fixed at $\sim 1.4$ times the absolute average deviation, as obtained by averaging over the complete non-seizure e-data, and was used in all the analyses as the reference scale length for all three data epochs. Smaller values for this scale length caused numerical problems in the determinations of the correlation dimension and the K-entropy; larger values limited the resolution of the nonlinear measures.

The clinical seizure in dataset \#73317 occurred from 53 to 95 s in the (third) seizure epoch (1253 to $1295 \mathrm{~s}$ in Figs. 5.1-5.4). Rhythmic convulsions began at 1295s, and post-ictal features appeared at $1314 \mathrm{~s}$. Table 5.1 shows features in the nonlinear measures that uniquely indicate the seizure; starred entries (*) denote no clear indicators. Two measures (the peak in $\mathrm{K}$ of $\mathrm{g}$-data and the peak in $D$ of h-data) show epilepsy onset beginning $\sim 30$ seconds before the clinical seizure.

Table 5.1 Summary of seizure indicators in dataset \#73317

\begin{tabular}{lllll}
\hline Specific measure & e-data & f-data & g-data & h-data \\
\hline $\begin{array}{l}\text { Time per cycle }\left(\mathrm{T}_{\mathrm{c}}\right) \\
\text { (timesteps per cycle) }\end{array}$ & $*$ & valley & $*$ & $*$ \\
& & $\mathrm{~T}_{\mathrm{c}}<200$ & & \\
& & $1255-1300 \mathrm{~s}$ & & \\
Entropy (K) & peak & 3 peaks & peak & peak \\
(bits/second) & $\mathrm{K}>0.063$ & $\mathrm{~K}>0.008$ & $\mathrm{~K}>0.056$ & $\mathrm{~K}>0.016$ \\
& $1230-1350 \mathrm{~s}$ & $1230-1240 \mathrm{~s}$ & $1220-1340 \mathrm{~s}$ & $1230-1320 \mathrm{~s}$ \\
& & $1270-1275 \mathrm{~s}$ & & \\
& & $1280-1300 \mathrm{~s}$ & & \\
& & & & \\
$1^{\text {st Min. in MIF (M) }}$ & $*$ & valley & $*$ & valley \\
(timesteps) & & $\mathrm{M}_{1}<85$ & & $\mathrm{M}_{1}<20$ \\
& & $1290-1310 \mathrm{~s}$ & & $1270-1280 \mathrm{~s}$ \\
& & & & \\
Correlation dimension (D) & peak & peak & peak & peak \\
& $\mathrm{D}>5$ & $\mathrm{D}>5.6$ & $\mathrm{D}>3.2$ & $\mathrm{D}>2$ \\
& $1270-1305 \mathrm{~s}$ & $1340-1345 \mathrm{~s}$ & $1235-1340 \mathrm{~s}$ & $1220-1325 \mathrm{~s}$ \\
\hline
\end{tabular}

Other trends in the average and standard deviation (SD) of each measure for dataset \#73317 are very different during the transition dataset (610-1190s) as compared to the non-seizure dataset (10-590s). We use the following notation for the subsequent discussion. The average of each measure is denoted by an under-barred symbol (e.g., the average entropy as $\mathrm{K}$; and the average correlation dimension as $\underline{\mathrm{D}}$ ). The SD of each measure $(\mathrm{x})$ is denoted by $\sigma_{\mathrm{x}}$ (e.g., the SD of the entropy as $\sigma_{K}$; and the SD of the correlation dimension as $\sigma_{D}$ ). 
The linear and nonlinear measures for dataset \#73317 have much variability, but clear distinctions exist between the non-seizure and transition results, as possible longer term predictors of seizure for this patient. For example, the SD in the skewedness $\left(\sigma_{z}\right)$ of $f$-data is $\geq 0.5$ for $440 \mathrm{~s}$ during transition (690-1130s), but remains at this level for only 200 s during the non-seizure period (60-260s). This same trend occurs in the e-data for 380s during transition (750-1130s), compared to 165s during the non-seizure period (80-245s). Thus, the continuous occurrence of $\sigma_{s} \geq 0.5$ for $>200$ s may be an indicator of an impending seizure for this patient.

A second trend in the transition data of dataset \#73317 (but not in the non-seizure data) is a regular sequence of quasi-periodic maximum and minima in $\underline{\mathrm{K}}$ and $\mathrm{D}$ of e-data at $710 \mathrm{~s}$ (max), $805 \mathrm{~s}(\mathrm{~min}), 900 \mathrm{~s}(\max ), 980 \mathrm{~s}(\mathrm{~min})$, and $1070 \mathrm{~s}(\max )$. The first four times are coincident with minima and maxima in $\underline{T}_{c}$ of e-data at $710 \mathrm{~s}(\mathrm{~min}), 805 \mathrm{~s}(\mathrm{max}), 900 \mathrm{~s}(\mathrm{~min})$, and $980 \mathrm{~s}(\mathrm{max})$. This trend also occurs in $\underline{\mathrm{K}}$ and $\underline{\mathrm{D}}$ of g-data at $690 \mathrm{~s}(\mathrm{max}), 790 \mathrm{~s}(\mathrm{~min}), 880 \mathrm{~s}(\mathrm{max}), 965 \mathrm{~s}(\mathrm{~min})$, and $1060 \mathrm{~s}$ (max), with corresponding minima and maxima in $\underline{T}_{c}$ for the first four times. This trend occurs again in $\underline{K}$ and $\underline{D}$ of h-data at $695 \mathrm{~s}(\max ), 790 \mathrm{~s}(\min ), 870 \mathrm{~s}(\max ), 960 \mathrm{~s}(\min )$, and 1060s (max), with corresponding minima and maxima in $\underline{T}_{c}$ that approximate the first four times. The times at which the extrema occur is within \pm 10 s among to $\mathrm{e} / \mathrm{g}-/ \mathrm{h}$-data, and is consistent with uncertainty that is inherent in the 10s overlap of successive analysis windows.

A third trend in the g-data of dataset \#73317 involves a valley in $\underline{M}_{1}$ that is coincident with a peak in $\sigma_{\mathrm{M}}$. The rise in $\sigma_{\mathrm{M}}$ begins at $785 \mathrm{~s}$, peaking at $885 \mathrm{~s}$, with the subsequent decrease ending at 990 s. The corresponding times for $\underline{M}_{1}$ (i.e., beginning of the fall, minimum, and end of the rise) are coincident with the corresponding time values for $\sigma_{\mathrm{M}}$. This trend in $\underline{M}_{1}$ and $\sigma_{\mathrm{M}}$ corresponds to oscillations in $M_{1}$ that increase in amplitude from 785-885s and subsequently decay from 885990 s. Note that the extrema in $\underline{M}_{1}$ and $\sigma_{M}$ occur at $885 \mathrm{~s}$, which is approximately coincident with one of the extrema times in $\left(\underline{K}, \underline{D}, \underline{T}_{c}\right)$ as discussed in the previous paragraph.

We infer other trends in dataset \#73317 from the total variation in the average and in the standard deviation of each measure during the non-seizure period relative to that during the transition period. For example, the average skewedness in e-data (see Fig. 5.1) is $-0.52 \leq \mathbf{s} \leq 0.25$ (during the non-seizure period) and $-0.51 \leq \underline{\mathrm{s}} \leq 0.24$ (during transition). The resulting total variations $(\Delta)$ in average are $\Delta_{n}=0.77$ (with the subscript " $n$ " denoting non-seizure) and $\Delta_{\mathrm{t}}=$ 0.75 (with the subscript " $\mathrm{t}$ " denoting transition). The ratio of these variations is $\Delta_{\mathrm{r}} / \Delta_{\mathrm{n}}=0.97$. Likewise, the total variation in the SD of the skewedness of e-data is $\Delta \sigma_{n}=0.9$ (non-seizure) and $\Delta \sigma_{\mathrm{r}}=0.6$ (transition). Then, the ratio of the total variation in standard deviation is $\Delta \sigma_{\mathfrak{l}} / \Delta \sigma_{\mathrm{n}}=$ 0.67 . Table 5.2 summarizes these comparisons between non-seizure and transition data, taken from Figs. 5.1-5.4. Most of these variations are not significant because noise in the EEG data (and biological data in general) makes comparisons tenuous for ratios near unity. However, starred values $\left({ }^{*}\right)$ in Table 5.2 are probably significant, corresponding to substantial ratios (e.g. $>2$ or $<0.5$ ). We note that some small ratios $(\leq 0.1)$ exist only for the linear measures of g-data (e.g., $\Delta_{\mathrm{r}} / \Delta_{\mathrm{n}}=0.016$ and $\Delta \sigma_{\mathrm{r}} / \Delta \sigma_{\mathrm{n}}=0.082$ for kurtosis, and $\Delta \sigma_{\mathrm{l}} / \Delta \sigma_{\mathrm{n}}=0.11$ for skewedness). In this patient, these variations mean that the non-seizure EEG is much more variable than the transition EEG for g-data for this type of seizure, and the transition is detectable with linear statistics. 
Table 5.2 Ratios of averages and standard deviations for dataset $\# 73317$

\begin{tabular}{cllll}
\hline Specific measure & e-data & f-data & g-data & h-dat \\
\hline skewedness (s) & & & & \\
$\Delta_{\sqrt{ }} \Delta_{\mathrm{n}}$ & 0.97 & 1.1 & $0.29^{*}$ & 0.77 \\
$\Delta \sigma_{\mathrm{J}} / \Delta \sigma_{\mathrm{n}}$ & 0.67 & $0.48^{*}$ & $0.11^{*}$ & 0.73
\end{tabular}

kurtosis $(\mathrm{k})$

$\begin{array}{lllll}\Delta_{\mathrm{l}} / \Delta_{\mathrm{n}} & 0.37^{*} & 0.90 & 0.016^{*} & 0.44^{*} \\ \Delta \sigma_{\mathrm{l}} / \Delta \sigma_{\mathrm{n}} & 0.28^{*} & 0.58 & 0.082^{*} & 0.42^{*}\end{array}$

Time per cycle $\left(T_{c}\right)$

$\begin{array}{lllll}\Delta_{\mathrm{l}} / \Delta_{\mathrm{n}} & 0.51 & 0.67 & 0.35^{*} & 0.63 \\ \Delta \sigma_{\mathrm{r}} / \Delta \sigma_{\mathrm{n}} & 0.67 & 0.62 & 0.35^{*} & 0.46^{*}\end{array}$

Entropy (K)

$\begin{array}{lllrl}\Delta_{\mathfrak{r}} / \Delta_{\mathrm{n}} & 0.71 & 0.50 & \ddots 0.70 & 0.53 \\ \Delta \sigma_{\mathrm{r}} / \Delta \sigma_{\mathrm{n}} & 0.93 & 0.33^{*} & 0.82 & 0.42^{*}\end{array}$

$1^{\text {st }}$ Min. in MIF $\left(M_{1}\right)$
$\Delta_{\mathrm{l}} / \Delta_{\mathrm{n}}$
1.3
$0.40^{*}$
0.52
0.67
$\Delta \sigma_{\mathfrak{l}} / \Delta \sigma_{\mathrm{n}}$
0.51
$0.40^{*} \div 0.91$
0.50

Correlation dimension (D)
$\Delta_{\mathrm{l}} / \Delta_{\mathrm{n}}$
0.56
0.73
$0.39 *$
0.97
$\Delta \sigma_{\mathrm{l}} / \Delta \sigma_{\mathrm{n}}$
0.64
0.57
0.50
0.78

We also analyzed portions of dataset \#73317 via the linear techniques described by Barlow [4]. Let $y_{i}$ be the sampled EEG signal with zero mean, so $y_{i}=e_{i}-\underline{e}$, with e determined from Eq. 3.1 The first parameter is the absolute mean value of the sampled signal (A):

$$
A=(1 / w) \sum_{i=1}^{w} L P\left(\left|y_{i}\right|\right)
$$

Here, $L P$ is a low-pass filter function with a time constant of $0.1 s$. The average value is determined over a window length of $\Delta t=0.5 \mathrm{~s}$ ( 256 data points). The second parameter is the absolute mean derivative of the sampled signal $\left(A^{\prime}\right)$ :

$$
A^{\prime}=(1 / w) \sum_{i=1}^{w} L P\left(\left|y_{i+1}-y_{i}\right| / \Delta t\right)
$$


The running mean curvature ( $\left.\mathrm{A}^{\prime \prime}\right)$ is obtained from an estimate of the absolute second derivative:

$$
A^{\prime \prime}=(1 / w) \sum_{i=1}^{w} L P\left(\left|y_{i+2}-2 y_{i+1}+y_{i}\right| /(\Delta t)^{2}\right)
$$

The running mean frequency $(F)$ is:

$$
\mathrm{F}=\mathrm{A}^{\prime} / \mathrm{A}
$$

The spectral purity index $(\mathrm{P})$ is:

$$
\mathrm{P}=\mathrm{FA} / \mathrm{A}^{\prime \prime}=\mathrm{A}^{\prime} / \mathrm{A}^{\prime \prime} \text {. }
$$

We applied these linear measures to e-data for portions of the non-seizure, transition, and seizure data segments. The results were unable to indicate the seizure period, nor even distinguish among the three segments (non-seizure, transition, and seizure). Details of the results are available from the authors on request. We devoted no further effort to this line of analysis.

The results from dataset \#73305 for channel 13 involved four analyses (e-, f-, g-, and h-data) on one 23-minute dataset that included all three epochs of channel 13 data (epileptic seizure, nonseizure, and transition from non-seizure to seizure). The non-seizure period spanned 10-400s. The transition period occurred over 410-1200s. The seizure began at 1245s and ended at 1290s. The patient was aphasic at $1300 \mathrm{~s}$, with head movements and verbalization at $1315 \mathrm{~s}$. The scale length $\left(\mathrm{L}_{0}\right)$ was fixed at $\sim 1.0$ times the absolute average deviation, as obtained by averaging over the non-seizure e-data, and was used in all the analyses as the reference scale length for all three data epochs. The results were obtained as before, and are plotted for e-data (Fig. 5.5), f-data (Fig. 5.6), g-data (Fig. 5.7), and h-data (Fig. 5.8). The seizure is indicated clearly by several unique features in the nonlinear measures, as shown in Table 5.3; starred entries $\left(^{*}\right)$ denote no clear indicators. None of these measures show the epilepsy onset before the clinical seizure.

A predictive trend in dataset \#73305 involves the sample standard deviation of the correlation dimension $\left(\sigma_{D}\right)$ for e-h data. In particular, $\sigma_{D}$ has a small variation during the non-seizure period, but undergoes large rises and falls during transition. Moreover, $\sigma_{D}$ has minimal variation $\left(\sigma_{D} \leq\right.$ 0.1 ) for $30-150 \mathrm{~s}$ intervals during transition. This trend is unique to the transition period.

A second predictive trend in dataset \#73305 involves the entropy $(\mathrm{K})$ for $f-$ and $h$-data. In these cases, $\mathrm{K}$ has a large, aperiodic variations during transition, and substantially less variation during the non-seizure period. Also during transition, the entropy becomes very small ( $\mathrm{K}<$ 0.00005 ) for several intervals that have a duration of $10-100$ s.

A third trend in dataset \#73305 occurs in the first minimum of the MIF $\left(M_{1}\right)$. From 700-1100s, $\underline{\mathrm{M}}_{1}$ in the e-data gradually decreases to a minimum of 21 just before the seizure. A similar decrease occurs in $\underline{M}_{1}$ from $400-1100$ s in g-data, to a minimum of 17 . Two cycles of a quasiperiodic variation of $\underline{M}_{1}$ in $f$-data appear from $400-800$ s, with a valley-to-valley period of 200 s. 

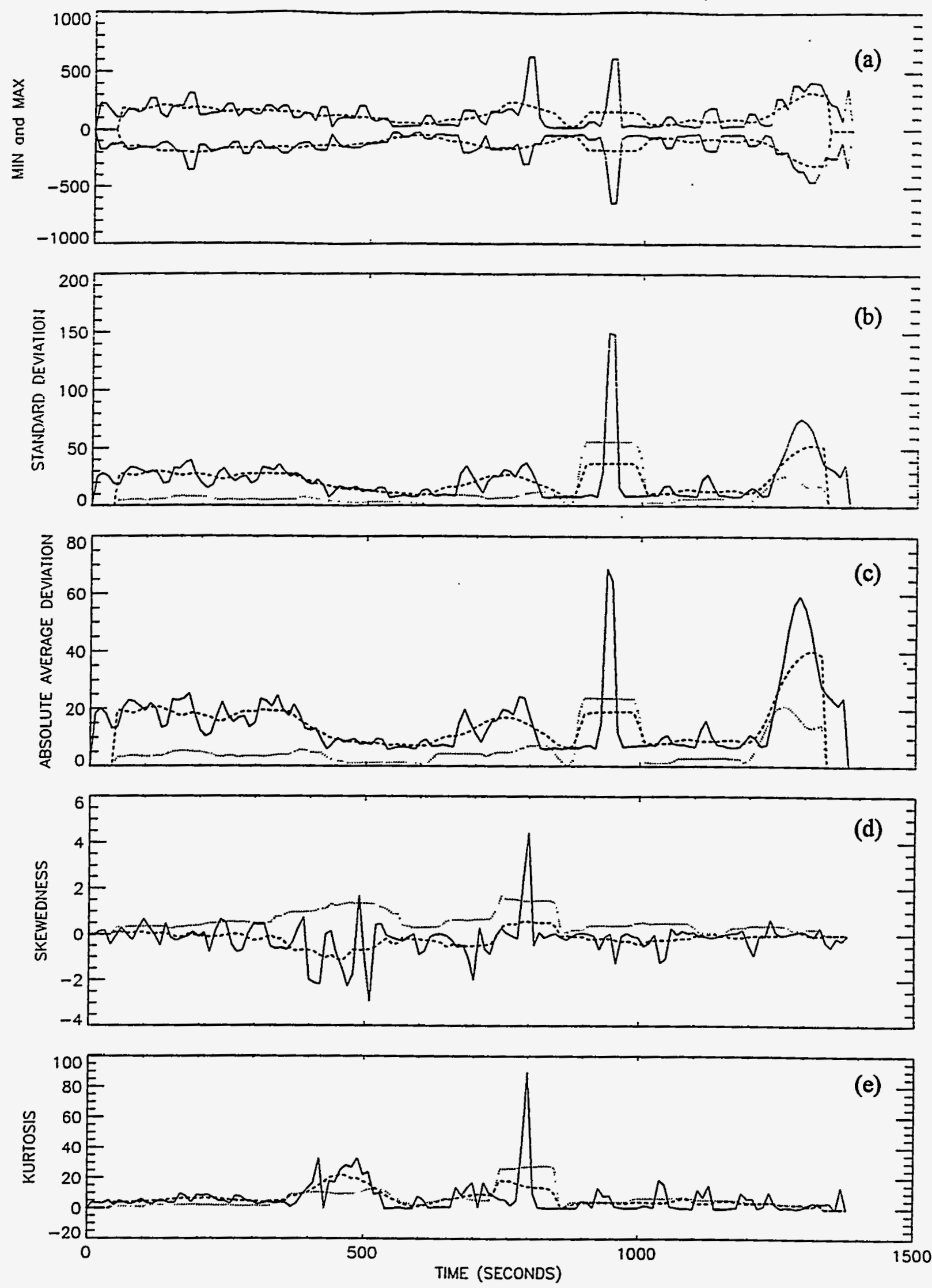

Fig. 5.5. Results for raw EEG (e-) data for dataset \#73305. The solid line is the specific measure, with the 11-point average (---) and standard deviation (...) of the measure. 

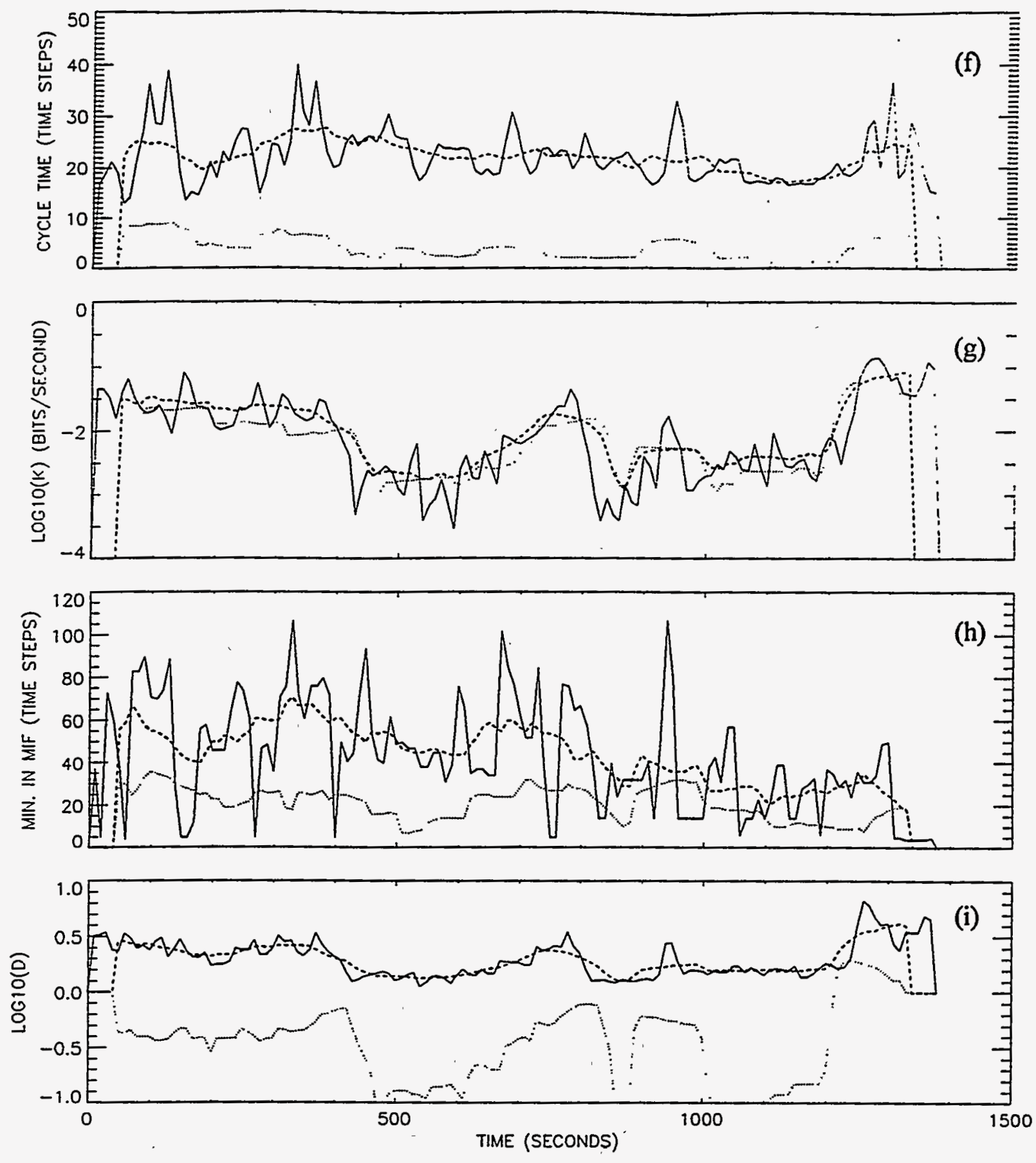

Fig. 5.5. (continued) 

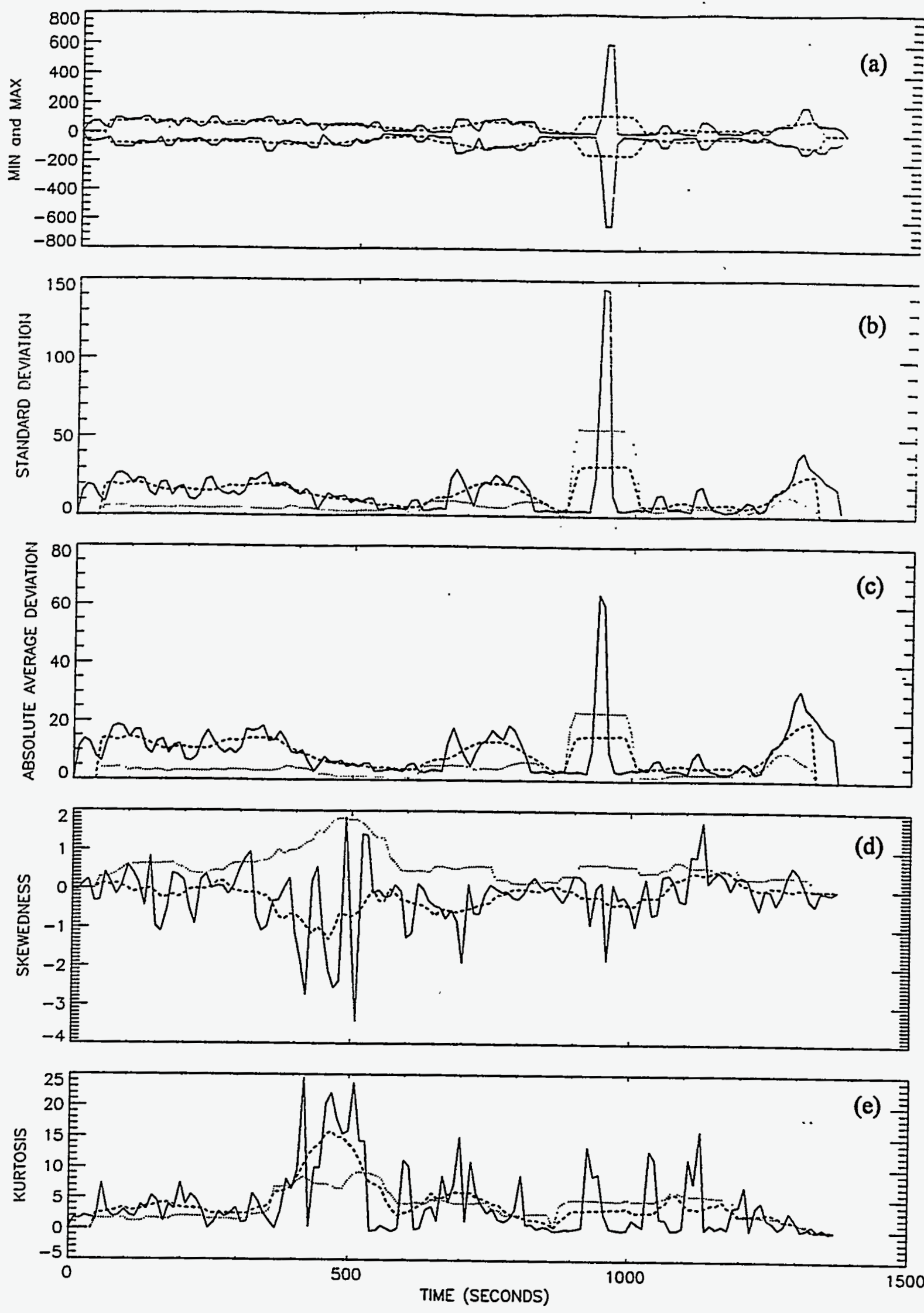

Fig. 5.6. Results for artifact EEG (f)- data for dataset \#73305. The solid line is the specific measure, with the 11-point average (- - -) and standard deviation (...) of the measure. 

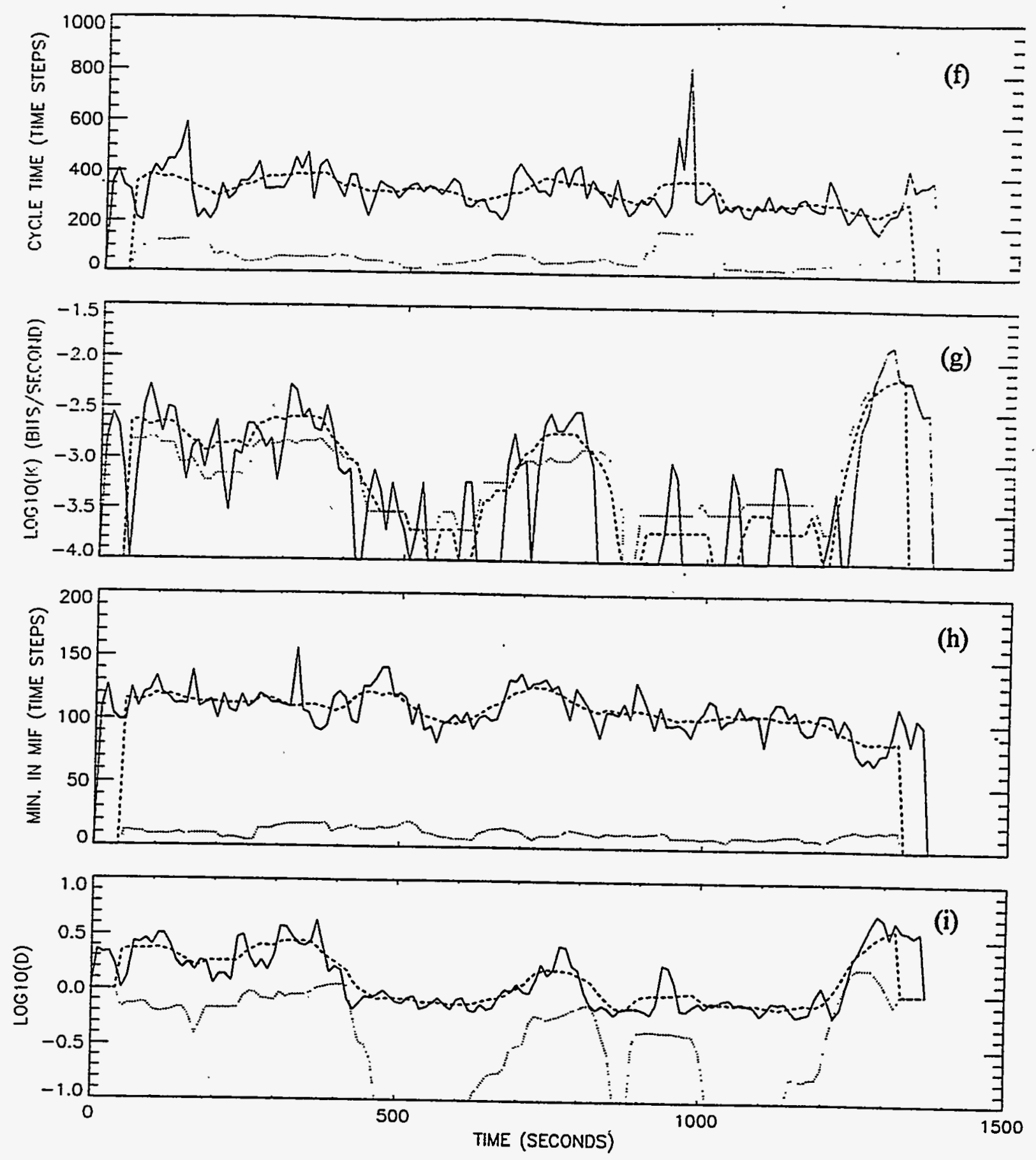

Fig. 5.6. (continued) 

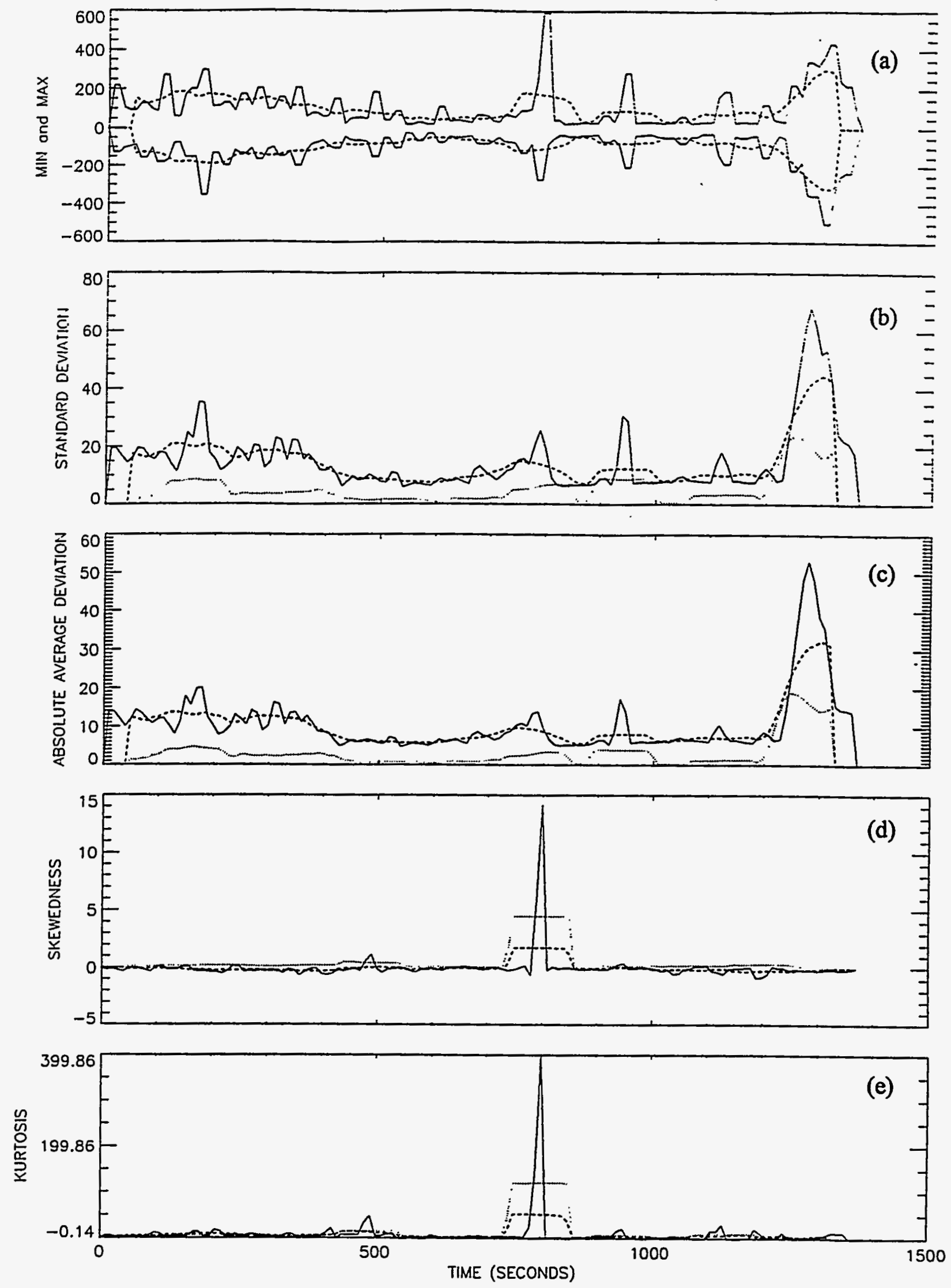

Fig. 5.7. Results for artifact-filtered EEG (g-) data for dataset \#73305. The solid line is the specific measure, with the 11-point average (---) and standard deviation (...) of the measure. 

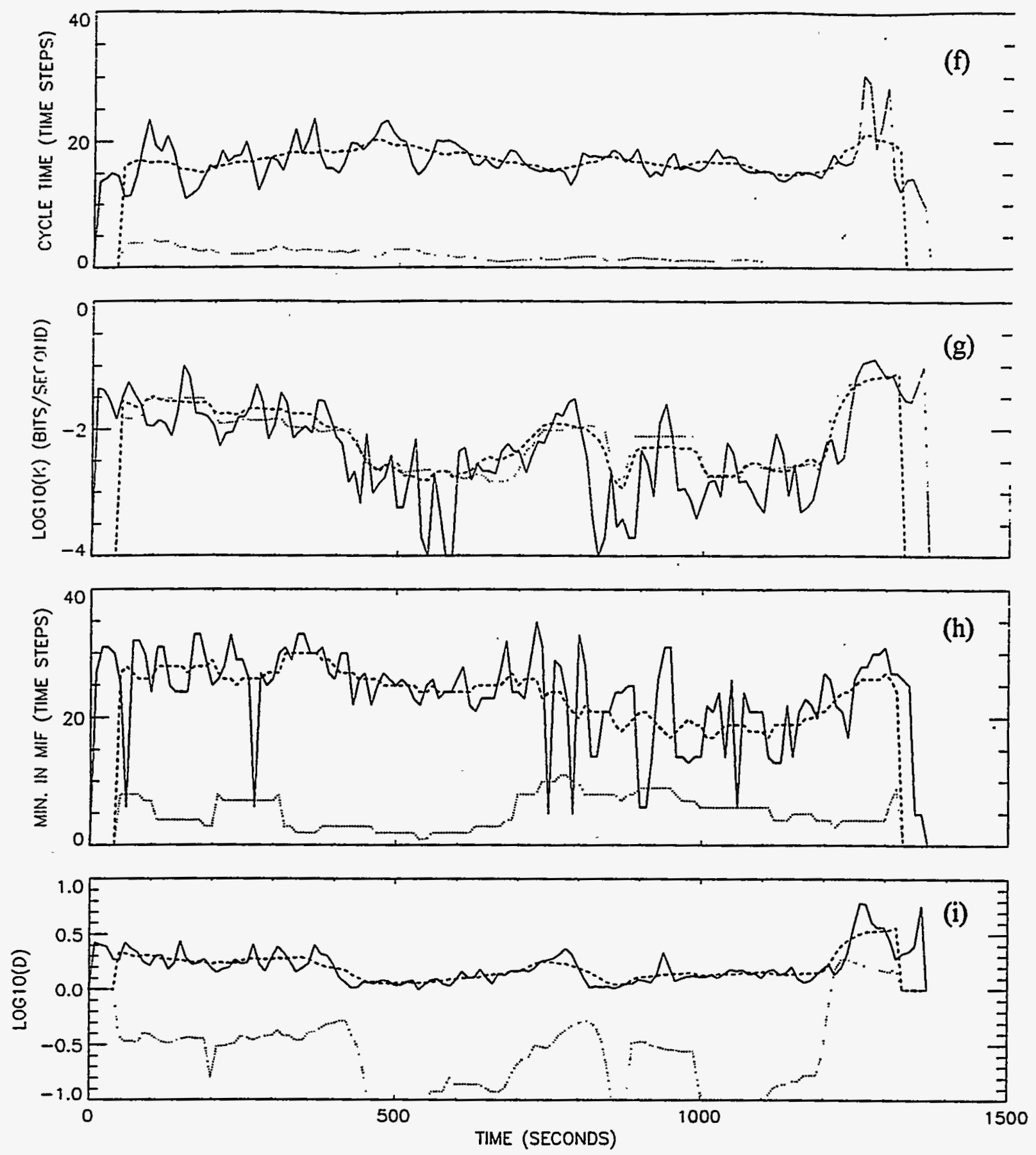

Fig. 5.7. (continued) 

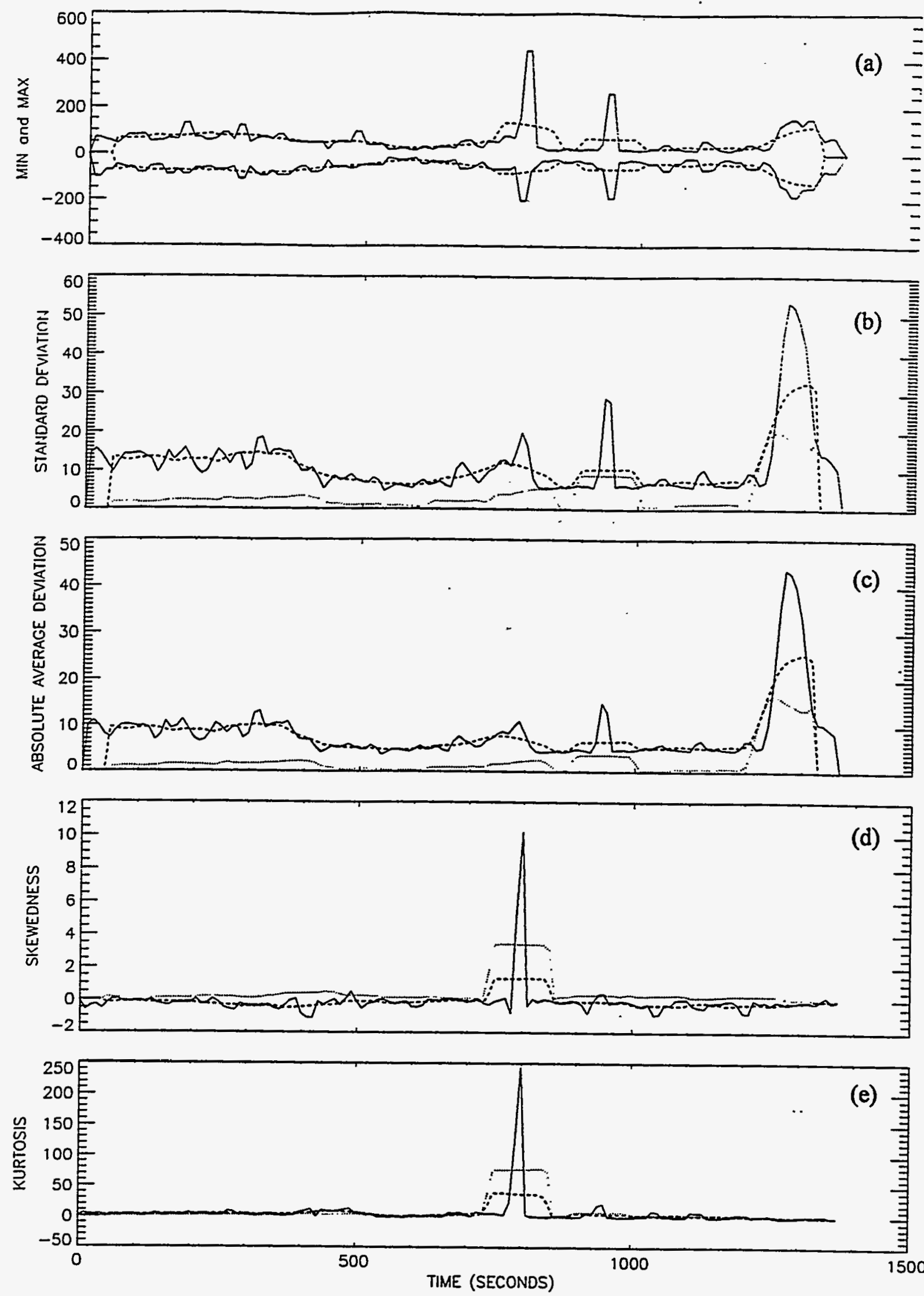

Fig. 5.8. Results for artifact- and low-pass-filtered EEG (h-) data for dataset \#73305. The solid line is the specific measure, with the 11-point average (-- $)$ and standard deviation (...) of the measure. 

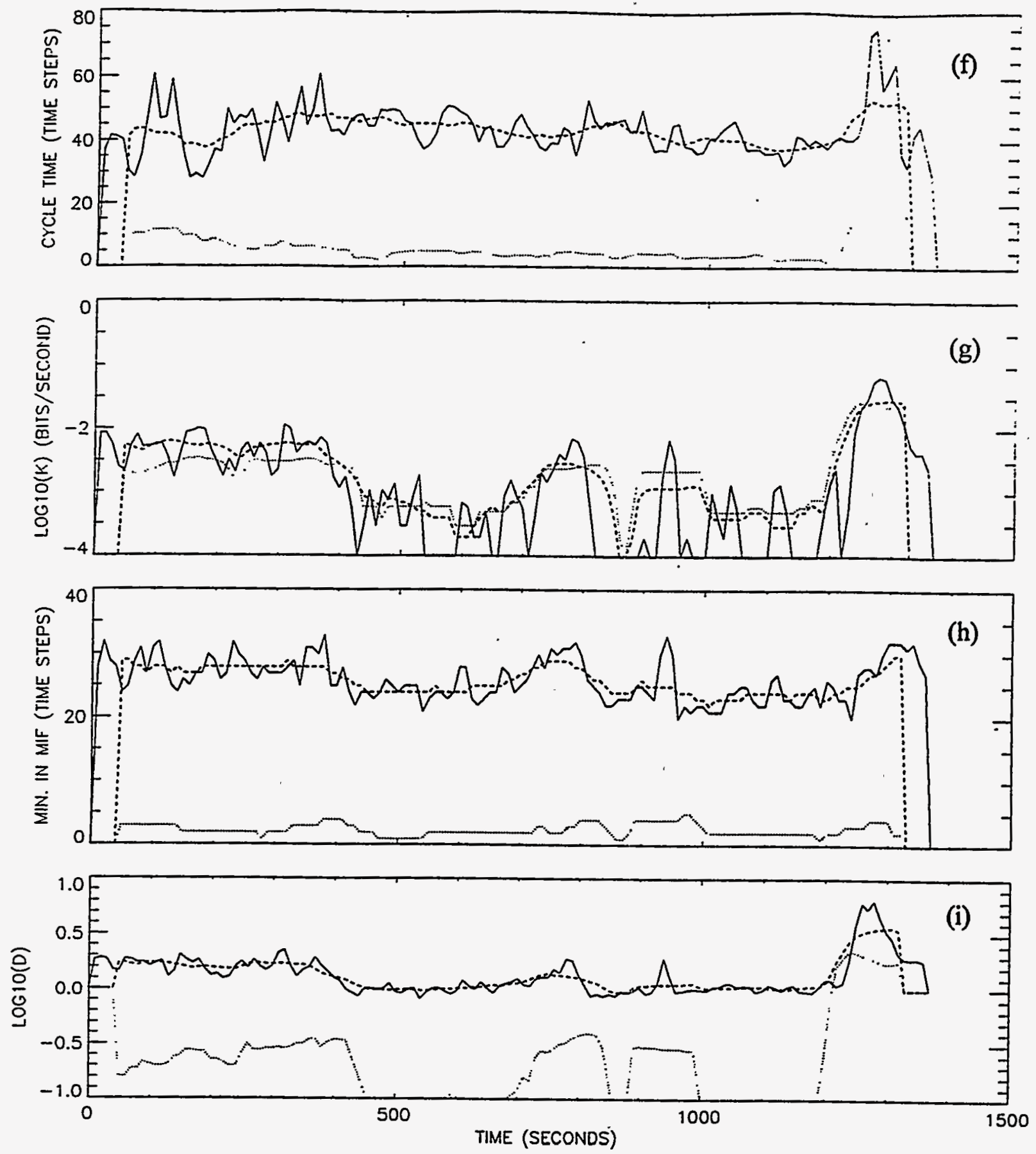

Fig. 5.8. (continued) 
Table 5.3 Summary of seizure indicators in dataset $\# 73305$

\begin{tabular}{|c|c|c|c|c|}
\hline Specific measure & e-data & f-data & g-data & h-data \\
\hline $\begin{array}{l}\text { Time per cycle }\left(T_{c}\right) \\
\text { (timesteps/cycle) }\end{array}$ & $*$ & $*$ & $\begin{array}{l}2 \text { peaks } \\
T_{c}>24 \\
1255-1275 \mathrm{~s} \\
1290-1300 \mathrm{~s}\end{array}$ & $\begin{array}{l}2 \text { peaks } \\
\mathrm{T}_{\mathrm{c}}>62 \\
1250-1275 \mathrm{~s} \\
1290-1300 \mathrm{~s}\end{array}$ \\
\hline $\begin{array}{l}\text { Entropy (K) } \\
\text { (bits/second) }\end{array}$ & $\begin{array}{l}\text { peak } \\
\mathrm{K}>0.089 \\
1255-1290 \mathrm{~s}\end{array}$ & $\begin{array}{l}\text { peak } \\
\mathrm{K}>0.006 \\
1270-1330 \mathrm{~s}\end{array}$ & $\begin{array}{l}\text { peak } \\
K>0.1 \\
1255-1290 \text { s }\end{array}$ & $\begin{array}{l}\text { peak } \\
\mathrm{K}>0.014 \\
1250-1320 \text { s }\end{array}$ \\
\hline $\begin{array}{l}1^{\mathrm{sl}} \text { Min. in MIF }\left(\mathrm{M}_{1}\right) \\
\text { (timesteps) }\end{array}$ & * & $\begin{array}{l}\text { valley } \\
M_{1}<80 \\
1250-1300 \text { s }\end{array}$ & $*$ & $*$ \\
\hline Correlation dimension (D) & $\begin{array}{l}\text { peak } \\
D>4 \\
1250-1300 \text { s }\end{array}$ & $\begin{array}{l}\text { peak } \\
D>4.5 \\
1280-1300 \mathrm{~s}\end{array}$ & $\begin{array}{l}\text { peak } \\
D>3 \\
1240-1310 \text { s }\end{array}$ & $\begin{array}{l}\text { peak } \\
D>2.5 \\
1240-1315 \mathrm{~s}\end{array}$ \\
\hline
\end{tabular}

A fourth trend in dataset \#73305 involves the entropy $(K)$ for e-data. From $600-730$ s, the . average entropy $(\underline{K})$ increases monotonically, while $K$ undergoes oscillations of decreasing amplitude about $\underline{\mathrm{K}}$. The entropy then decreases abruptly $(\mathrm{K}=0.045-\mathrm{K}=0.0004)$ during 760 820 s. Then, $\mathrm{K}$ again increases monotonically over $850-920 \mathrm{~s}$, while $\mathrm{K}$ undergoes oscillations of increasing amplitude about this average. The entropy then decreases abruptly $(\mathrm{K}=0.018-$ $\mathrm{K}=0.001$ ) during $930-970 \mathrm{~s}$. The previous cycle is repeated over 1000-1100s (increasing $\underline{\mathrm{K}}$ with $\mathrm{K}$ undergoing oscillations of increasing amplitude), followed by a smaller decrease in ( $\mathrm{K}=0.009-$ $\mathrm{K}=0.003$ ). This last decrease is hard to distinguish from the imbedded oscillations, which subsequently rise again as part of the seizure.

A fifth trend in dataset \#73305 entails the variability in skewedness and kurtosis of $\mathrm{f}$-data. Figure 5.6 shows that from $0-350$ s, skewedness and kurtosis have moderate variability (with small sample standard deviations), which increases markedly during 350-550s (with large values for the sample standard deviations). Subsequently, the skewedness returns to moderate values, while the sample standard deviation of the kurtosis remains at 2-3 times the non-seizure value.

Other trends in the average and standard deviation of each measure for dataset \#73305 are very different during the transition period (410-1200s) as compared to the non-seizure period (10$400 \mathrm{~s}$ ). Table 5.4 shows the ratios of the variations in the average and in the standard deviation of each measure during the non-seizure period with that during the transition period. As before, most of these variations are not significant because noise in the EEG data makes comparisons tenuous for ratios near unity. However, starred values $\left({ }^{*}\right)$ in Table 5.4 are probably significant, 
corresponding to substantial ratios (e.g. $>2$ or $<0.5$ ). We note that some large ratios $(>10)$ occur for the linear measures in Table 5.4. These large ratios mean that the transition data is much more variable for this seizure than for the non-seizure data.

Table 5.4 Ratios of averages and standard deviations for dataset \#73305

\begin{tabular}{cllll}
\hline Specific measure & e-data & f-data & g-data & h-data \\
\hline std. deviation $(\sigma)$ & & & & \\
$\Delta_{\mathrm{r}} / \Delta_{\mathrm{n}}$ & $3.9^{*}$ & $4.8^{*}$ & $2.1^{*}$ & $3.3^{*}$ \\
$\Delta \sigma_{\mathrm{l}} / \Delta \sigma_{\mathrm{n}}$ & $15.7^{*}$ & $25.9^{*}$ & 1.2 & $6.5^{*}$
\end{tabular}

abs. avg. dev. (a)

$\begin{array}{lllll}\Delta_{\mathrm{r}} / \Delta_{\mathrm{n}} & 2.5^{*} & 2.9^{*} & 2.3^{*} & 3.0^{*} \\ \Delta \sigma_{\mathrm{r}} / \Delta \sigma_{\mathrm{n}} & 10.6^{*} & 12.7^{*} & 1.1 & 3.6^{*}\end{array}$

skewedness ( $s$ )

$\begin{array}{lllll}\Delta / \Delta \Delta_{\mathrm{n}} & 4.8^{*} & 4.1^{*} & -8.6^{*} & 5.5^{*} \\ \Delta \sigma_{\mathrm{r}} / \Delta \sigma_{\mathrm{n}} & 3.1^{*} & 3.8^{*} & 44.5^{*} & 23.9^{*}\end{array}$

kurtosis (k)

$\begin{array}{lllll}\Delta_{\mathrm{r}} / \Delta_{\mathrm{n}} & 6.9^{*} & 8.6^{*} & 10.8^{*} & 17.5^{*} \\ \Delta \sigma_{\mathfrak{l}} / \Delta \sigma_{\mathfrak{n}} & 11.7^{*} & 6.9^{*} & 36.1^{*} & 54.2^{*}\end{array}$

Time per cycle $\left(\mathrm{T}_{\mathrm{c}}\right)$

$\begin{array}{lllll}\Delta / \Delta_{\mathrm{n}} & 1.4 & 1.3 & 1.8 & 1.0 \\ \Delta \sigma_{\mathfrak{r}} / \Delta \sigma_{\mathrm{n}} & 1.2 & 1.8 & 1.0 & 0.67\end{array}$

Entropy (K)

$\begin{array}{lllll}\Delta / \Delta_{\mathrm{n}} & 1.5 & 1.9 & 1.00 & 2.0 \\ \Delta \sigma_{\mathfrak{r}} / \Delta \sigma_{\mathrm{n}} & 0.99 & 1.5 & 0.49^{*} & 1.6\end{array}$

$1^{\text {st }}$ Min. in MIF $\left(M_{1}\right)$

$\begin{array}{lllll}\Delta \sqrt{ } \Delta_{\mathrm{n}} & 1.5 & 3.0^{*} & 2.6^{*} & 3.5^{*} \\ \Delta \sigma_{\mathrm{r}} / \Delta \sigma_{\mathrm{n}} & 1.4 & 1.1 & 1.7 & 2.0\end{array}$

Correlation dimension (D)

$\begin{array}{llllll}\Delta / \Delta_{\mathrm{n}} & 1.8 & 1.8 & & 1.7 & 3.3^{*} \\ \Delta \sigma_{\mathrm{r}} / \Delta \sigma_{\mathrm{n}} & 4.3^{*} & 1.9 & & 1.7 & 2.2^{*}\end{array}$




\section{CONCLUSIONS}

We analyzed two sets of channel-13 EEG data from one patient. Both sets of data included non-seizure, transition-to-seizure, and epileptic seizure data. The analysis included various linear measures (standard deviation, absolute average deviation, skewedness, kurtosis), plus nonlinear measures (time steps per cycle, Kolmogorov entropy, first minimum in the mutual information function, and correlation dimension). We analyzed four forms of the data: raw EEG (e) data, artifact $(f)$ data via application of a zero-phase quadratic filter, artifact-filtered $(g)$ data that was the residual after subtracting f-data from g-data, and a low-pass-filtered version ( $h$ ) of the $g$-data. The nonlinear measures clearly discerned the epileptic seizures in this patient, but none of the linear measures provided a definitive seizure indication. Both linear and nonlinear measures provide trends that may predict seizure onset in this patient, but the trends are different for each seizure. Our nonlinear tools found seizure indicators and predictive trends with and without artifact removal, with and without low-pass filtering, demonstrating the robustness of these tools to noise and artifacts. Surrogate analysis of e-data from dataset \#73305 showed that this data has significant nonlinear structure.

For this patient, peaks in both the Kolmogorov entropy and correlation dimension indicate both epileptic seizures, although the seizures are clinically very different, as described at the beginning of Section 4. Valleys in $\left(T_{c}\right)$ number of timesteps per cycle (for $f$-data) and in $\left(M_{1}\right)$ the first minimum in the mutual information function (for $\mathrm{f}$ - and $\mathrm{h}$-data) also indicate the first seizure (dataset \#73317). A valley in $\mathrm{M}_{1}$ (f-data) and peaks in $\mathrm{T}_{\mathrm{c}}$ (g-and $\mathrm{h}$-data) mark the second seizure (dataset \#73305). These indicators of seizure are summarized in Tables 5.1 and 5.3. The difference in seizure indication $\left(T_{c}\right)$ for the two seizures implies that care is needed in cataloging such features.

Our analysis for this patient also reveals trends in linear and nonlinear measures that precede the seizure by 500-800 seconds, as shown in Figs. 5.1-5.8, and as discussed in the text. These trends are easily computable and may permit seizure prediction in this patient. Transition indicators are very different for the two seizures, so predictive trends must be chosen with care.

We emphasize that these results are based on the analysis of one channel of EEG data for two separate seizures in a single patient. Generalization of these results to other seizure types or to other patients is inappropriate. Consequently, much additional work is needed to establish a statistical basis for epilepsy prediction, e.g., by examining $\geq 10$ datasets from each of 10-15 patients. However, the positive indications of seizure detection and prediction is the present work provide a hypothesis upon which such a generalization may be possible. 


$$
\cdots 1
$$




\section{ACKNOWLEDGMENTS}

We gratefully acknowledge members of the staff at the Knoxville Neurology Clinic, including Dr. Ronald E. Leppanen, Walter W. Holland, and Helen L. Leach, who provided expert assistance with the EEG data (acquisition, selection, identification and classification of artifacts). The authors thank Dr. Vladimir A. Protopopescu and John Thomas for his helpful comments on our manuscript. We also thank Joyce B. Echols for assistance in preparing the manuscript.

This research was sponsored by the Laboratory Directed Research and Development Program of Oak Ridge National Laboratory, managed for the United States Department of Energy by Martin Marietta Energy Systems, Inc., under Contract No. DE-AC05-84OR21400. 


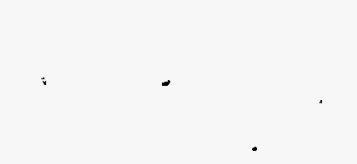




\section{REFERENCES}

1. A. Babloyantz, J. M. Salazar, and C. Nicolis, "Evidence of Chaotic Dynamics of Brain Activity During the Sleep Cycle," Phys. Lett. A 111, 152-156 (1985) .

2. A. Babloyantz and A. Destexhe, "Low-Dimensional Chaos in an Instance of Epilepsy," Proc. Natl. Acad. Sci. USA 83, 3513-3517 (1986).

3. A. Babloyantz and A. Destexhe, in From Chemical to Biological Organization, edited by M. Markus, S. Muller, and G. Nicolis, Springer-Verlag Publ., Berlin, 1988.

4. J. S. Barlow, The Electroencephalogram, Its Patterns and Origins, (A Bradford Book) MIT Press, Boston, 1993.

5. E. Basar (ed.), Chaos in Brain Function, Springer-Verlag Publ., Berlin, 1990.

6. M. L. Cartwright and J. W. Littlewood, J. London Math. Soc. 20, 180 (1945).

7. R. Coxeter, "Helping Epileptic Brains Find.Their Way Back to Chaos," Business Week 9/5/94 issue, pg. 83 .

8. A. Destexhe, J. A. Sepulchre, and A. Babloyantz, "A Comprehensive Study of the Experimental Quantification of Deterministic Chaos," Phys. Lett. A. 132, 101-106 (1988).

9. I. Dvorak and J. Siska, "On Some Problems Encountered in the Estimation of the Correlation Dimension of the EEG," Phys. Lett. A 118, 63-66 (1986).

10. I. Dvorak, "Takens versus Multichannel Reconstruction in EEG Correlation Exponent . Estimates," Phys. Lett. A 151, 225-233 (1990).

11. D. F. Elliott and K. R. Rao, Fast Transforms, Analyses, Applications, Academic Press, 1982.

12. G. W. Frank, T. Lookman, M. A. H. Nerenberg, C. Essex, J. Lemieux, and W. Blume, "Chaotic Time Series Analysis of Epileptic Seizures," Physica D 46,427-438 (1990).

13. A. M. Fraser and H. L. Swinney, "Independent Coordinates for Strange Attractors from Mutual Information," Phys. Rev A 33, 1134-1140 (1986).

14. J. Glanz, "Do Chaos-Control Techniques Offer Hope for Epilepsy?" Science 265, 1174 (1994). 
15. J. Gotman, "Seizure Recognition and Analysis," Long-term Monitoring in Epilepsy (EEG Suppl. No. 37), pgs. 133-145 (1985).

16. A. Gottschalk, M. S. Bauer, and P. C. Whybrow, "Long-term Organization of Mood in Bipolar Disorder," Dynamical Systems in the Neurosciences - Multiscale Time and Space Coherence in Brain Function (Satellite Symposium of the Annual Meeting of the Society for Neuroscience, Washington, D.C. (November, 1993).

17. G. T. Kenyon, "Using Asymptotic Kolmogorov Entropy to Identify Order in Spike Train Data," Dynamical Systems in the Neurosciences - Multiscale Time and Space Coherence in Brain Fumction (Satellite Symposium of the Annual Meeting of the Society for Neuroscience, Washington, D.C. (November, 1993).

18. G. A. Korn and T. M. Korn, Mathematical Handbook for Scientists and Engineers, McGraw-Hill Book Company, New York, 1968.

19. A. D. Krystal, "Spatio-Temporal Analysis of Electroconvulsive Therapy Seizures," Dynamical Systems in the Neurosciences - Multiscale Time and Space Coherence in Brain Function (Satellite Symposium of the Annual Mtg. of Soc. for Neuroscience, Washington, D.C. (November, 1993).

20. W. F. Lawkins, N. E. Clapp, C. S. Daw, L. M. Hively, and M. L. Eisenstadt, "An Analysis of Normal State EEG Data ...," to be published (1995).

21. N. Levinson, "A Second Order Differential Equation with Singular Solutions," Ann. Math. 50, 127 (1949).

22. A. J. Mandell and M. F. Shlesinger, in The Ubiquity of Chaos, edited by S. Krasner, AAAS Press, Washington, D.C., 1990.

23. G. Mayer-Kress and S. P. Layne, Dimensionality of the Human Electroencephalogram," Am. N.Y. Acad. Sci. 504, 62-87 (1987).

24. F. Moss, "Chaos under Control," Nature 370, 596-597 (1994).

25. D. E. Olsen, J. A. Cristion, and C. W. Spaur, "Automatic Detection of Epileptic Seizures Using Electroencephalographic Signals," Johns Hopkins APL Techn. Digest 12, 182-191 (1991).

26. E. Pennisi, "Seeing and Controlling Chaos in the Brain," Sci. News 146, 134 (1994).

27. J. P. M. Pijn, "Quantitative Evaluation of EEG Signals in Epilepsy - Nonlinear Associations, Time Delays, and Nonlinear Dynamics," Ph.D. Thesis, University of Amsterdam, 1990. 
28. J. P. Pijn, J. van Neerven, A. Noest, and F. H. Lopes da Silva, Electroencephal. Clinical Neurophysiol. 79, 371 (1991).

29. W. H. Press, B. P. Flannery, S. A. Teukolsky, and W. T. Vetterling, Numerical Recipes: The Art of Scientific Computing, Cambridge University Press, Cambridge, England, 1986.

30. D. Prichard and J. Theiler, "Generating Surrogate Data for Time Series with Several Simultaneously Measured Variables," Phys. Rev. Lett. 73, 951-954 (1994).

31. W. S. Pritchard and D. W. Duke, "Measuring Chaos in the Brain - A Tutorial Review of Nonlinear Dynamic EEG Analysis," Int. J. Neurosci. 67, 31-80 (1992).

32. L. R. Rabiner and B. Gold, Theory and Application of Digital Signal Processing, Prentice Hall Inc., 1975.

33. P. E. Rapp, "Dynamical Analysis of Biological Systems: A Case for Guarded Optimism," The Head and Heart of Chaos - Nonlinear Dynamics in Biological Systems, NIH workshop (June 1992).

34. P. E. Rapp, A. M. Albano, I. D. Zimmerman, and M. A. Jiménez-Montaño, "PhaseRandomized Surrogates Can Produce Spurious Identifications of Non-Random Structure," Phys. Lett. A 192, 27-33 (1994).

35. J. Röschke and J. Aldenhoff, "The Dimensionality of Human Electroencephalogram during Sleep," Biol. Cybern. 64, 307-313 (1991).

36. S. M. Selby and B. Girling, Standard Mathematical Tables, page 390, The Chemical Rubber Co. (14th edition, 1965).

37. S. J. Schiff, "Stochastic versus Deterministic Variability in Simple Neuronal Circuits," Dynamical Systems in the Neurosciences - Multiscale Time and Space Coherence in Brain Function (Satellite Symposium of the Annual Meeting of the Society for Neuroscience, Washington, D.C. (November, 1993)

38. S. J. Schiff, K. Jerger, D. H. Duong, T. Chang, M. L. Spano, and W. L. Ditto, "Controlling Chaos in the Brain," Nature 370, 615-620 (1994).

39. J. C. Schouten, F. Takens, and C. M. van den Bleek, "Maximum-Likelihood Estimation of the Entropy of an Attractor," Phys. Rev. E 49, 126-129 (1994).

40. J. C. Schouten, F. Takens, and C. M. van den Bleek, "Estimation of the Dimension of a Noisy Attractor," Phys. Rev. E 50, 1851-1861 (1994).

41. C. E. Shannon and W. Weaver, The Mathematical Theory of Communication, University of Illinois Press, Urbana, 1949. 
42. A. C. K. Soong and C. I. J. M. Stuart, "Evidence for Chaotic Dynamics Underlying the Human Alpha-Rhythm Electroencephalogram," Biol. Cybern.62, 55-62 (1989).

43. K. J. Stam, D .L. J. Tavy, B. Jelles, H. A. M. Achtereekte, J. P. J. Slaets, and R. W. M. Keunen, "Non-Linear Dynamical Analysis of Multichannel EEG: Clinical Applications in Dementia and Parkinson's Disease," Brain Topography 7, 141-150.(1994).

44. F. Takens, "On the Numerical Determination of the Dimension of an Attractor," in Dynamical Systems and Bifurcations, ed. B.L.J. Braaksma, H.W. Broer, and F. Takens, Lecture Notes in Mathematics 1125, 99-106 (1984) Springer-Verlag, Berlin.

45. F. J. Taylor, Digital Filter Design Handbook," Marcel Dekker, Inc., Publ., New York and Basel, 1983.

46. M. C. Teich, "Neuronal Firing Patterns with Multiscale Time Coherence," Dynamical Systens in the Neurosciences - Multiscale Time and Space Coherence in Brain Function, Satellite Symp. of the Annual Meeting of the Society for Neuroscience, Washington, D.C. (November, 1993)

47. J. Z. Tepper and A. J. Mandell, Amm. N.Y. Acad. Sci. 504, 88 (1987).

48. J. Theiler, "On the Evidence for Low-Dimensional Chaos in an Epileptic Electroencephalogram," Phys. Lett. A 196, 335-341 (1995).

49. R. C. Watt and S. R. Hameroff, "Phase Space Analysis of Human EEG during General Anesthesia," Amn. N.Y. Acad. Sci. 504, 286-288 (1987).

50. R. C. Watt and S. R. Hameroff, "Phase Space Electroencephalography (EEG): a New Mode of Intraoperative EEG Analysis," Int. J. Clin. Monit. Comput. 5, 3-13 (1988).

51. A. S. Weigend and N. A. Gershenfeld, "Time Series Prediction: Forecasting the Future and Understanding the Past, "Proceedings of the NATO Advanced Research Workshop on Comparative Time Series Analysis (Santa Fe, New Mexico, 14-17 May 1992) Addison-Wesley Publishing Company, 1994. 


\section{DISTRIBUTION}

M. A. Akerman

B. A. Berven

N. E. Clapp

W.K. Danenhart

C. S. Daw

D. J. Downing

P.C. Gailey

R. B. Gilliland

L. M. Hively

R. F. Holdaway

M. R. Kennel

W. F. Lawkins

R. W. Leggett

M. R. Leuze

G. T. Mays

G. W. Morrison

C. E. Oliver

V. A. Protopopescu

D. E. Reichle

R. M. Schilling, Jr.

R. M. Sincovec

T. P. Sjoreen

J. O. Stiegler

J. F. Thomas

B. E. Tonn

D. W. Walker

Laboratory Records - RC

Laboratory Records (2 copies)

Patent Office 